\begin{tabular}{|c|c|c|}
\hline \multirow{3}{*}{$\begin{array}{l}\text { ITC 4/48 } \\
\text { Information Technology } \\
\text { and Control } \\
\text { Vol. 48/ No. 4/ } 2019 \\
\text { pp. 557-578 } \\
\text { DOI 10.5755/j01.itc.48.4.23303 }\end{array}$} & \multicolumn{2}{|c|}{$\begin{array}{c}\text { Model Predictive Control of UCG: An Experiment } \\
\text { and Simulation Study }\end{array}$} \\
\hline & Received 2019/04/30 & Accepted after revision 2019/10/21 \\
\hline & \multicolumn{2}{|c|}{ Cross } \\
\hline
\end{tabular}

\title{
Model Predictive Control of UCG: An Experiment and Simulation Study
}

\section{Ján Kačur, Patrik Flegner, Milan Durdán, and Marek Laciak}

Faculty of Mining, Ecology, Process Control, and Geotechnologies; Institute of Control and Informatization of Production Processes, Technical University of Košice, Němcovej 3, 04001 Košice, Slovak Republic; e-mail:jan.kacur@tuke.sk

Corresponding author: jan.kacur@tuke.sk

Underground coal gasification (UCG) is a potential technology that enables to mine coal without traditional mining equipment. The coal is gasified deep in underground and produced syngas is processed on the surface. The most important technical problem in UCG is unstable quality of syngas and control. This paper proposes advanced control based on an adaptive predictive controller. The maintaining of desired calorific value depends on flow rates of gasification agents injected to the underground geo-reactor and controlled exhaust. The paper proposes a physical model of UCG technology and applies a method of multivariate adaptive regression splines (MARS) to model the gasification process. This method satisfactorily approximates nonlinearity in the process variables. The paper proposes adaptive model predictive control (MPC) using online model estimation and applied it on the MARS model of UCG that imitates the real process. The results have shown that optimization of manipulation variables can replace manual control in UCG. Getting better quality of syngas depends on setpoints, optimized manipulation variables, and constraints used in MPC. In simulations, the adaptive MPC has shown better performance in comparison with manual and PI control.

KEYWORDS:UCG, Coal, Gasification, Prediction, Model Predictive Control, MARS, Matlab-Simulink.

\section{Introduction}

Underground coal gasification (UCG) represents in-situ controlled combustion of coal where valuable gas (i.e., syngas) is produced. Conventional mining methods can extract only $15 \%$ of the total coal reserves. UCG offers an alternative to traditional mining of coal, especially in case of coal seams which are 
not mineable by conventional approach or have some tectonic failures. Moreover, it is less expensive than traditional mining. UCG has the potential to be used in the future. In in-situ gasification two wells drilled into underground are needed to initiate gasification, i.e., injection and production well. In UCG, heat is generated in the underground coal bed using injected oxidants (i.e., by endothermic chemical reactions). This heat is used by exothermic reactions with carbon to produce syngas components. With the need to improve the gasification process, it must be ensured that the combustive reactions have generated enough energy to heat of reactants. The calorific value of the produced syngas is the most important indicator of gasification quality. In UCG, various gasification agents are used, e.g., air, oxygen, or water vapor. In an underground reactor, the processes of drying, pyrolysis of solid hydrocarbon, combustion and gasification continually take place. UCG represents reaction zones defined by temperatures where the combustion front progressively moves. Groundwater also participates in gasification. Raw, pure syngas from UCG contains predominantly $\mathrm{CO}, \mathrm{CO}_{2}, \mathrm{H}_{2}, \mathrm{CH}_{4}$, and $\mathrm{O}_{2}$, higher hydrocarbons, tar, and impurities. Monitoring the underground temperature in the oxidation zone is important for the control of UCG. The UCG runs in temperatures close to $1000^{\circ} \mathrm{C}$ according to Figure 1. At the fire face, there are high temperatures (up to $1200-1300^{\circ} \mathrm{C}$ ). An extensive overview of UCG can be found in $[9,12,39]$.

\section{Figure 1}

Principle of the UCG with reaction zones

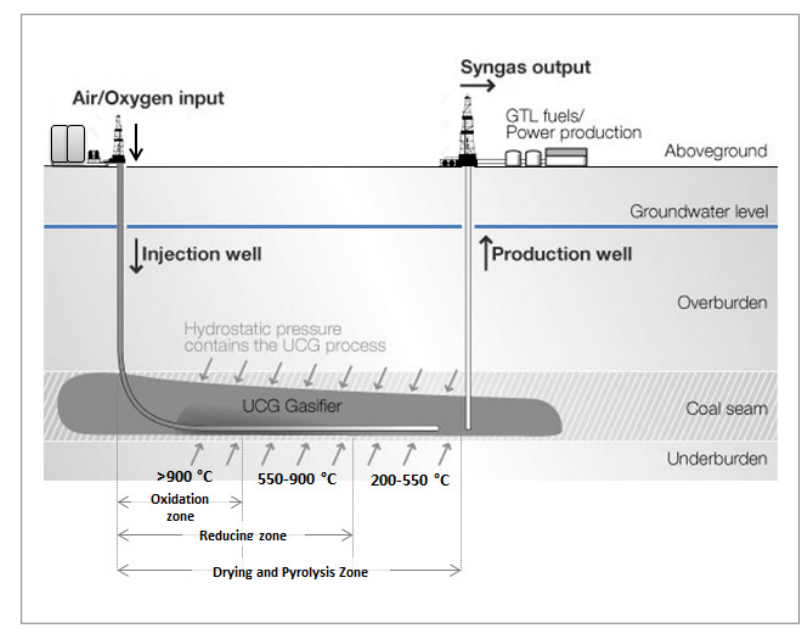

Research in the world is mainly focused on modeling internal processes of UCG and on model-based control. This paper examines the possibilities of model predictive control of UCG with adapted internal controller model. In this paper, the simulation study is performed to assess the suitability of the proposed approach. Major contributions of this paper are as follows: 1) A data-driven modeling of syngas calorific value and underground temperature by utilizing the model based on multivariate adaptive regression splines (MARS). The MARS model will be used to imitate the UCG process in simulations. Such an approach has not yet been applied in the world; 2) A proposal of the adaptive predictive control to maintain or increase the syngas calorific value. Such an approach to UCG control has not yet been verified. 3) A comparison of the proposed predictive control with manual control and classical PI control. The following subsections give an overview of recent research in the field of UCG modeling and control.

\subsection{UCG Modeling}

The UCG involves a complex range of physical processes occurring over a wide range of characteristic time and length scales. Modeling of such processes is a compromise between model complexity (often assumed to give better predictive capability) versus simplicity (known to provide faster computational runtimes). The tradeoffs made in the model development are typically a function of the model's intended purpose, expectations of the uncertainties, and sensitivities of model inputs the current state-of-knowledge and the available computing power.

A comprehensive UCG model should include various physical phenomena (e.g., heat and mass transfers, coal drying and pyrolysis, chemical reactions cavity evolution, liquid flow and interaction between surrounding environment) and physical sub-processes (e.g., cavity growth, combustion front propagation, the interaction of the cavity with overburden and hydrology) [45].

In the literature, different approaches to UCG modeling can be found. Earliest numerical models were one-dimensional packed bed $[40,57]$. Thorsness et al. [49] were able to make a good prediction of syngas composition and coal consumption for laboratory ex- 
periments with steam and oxygen (ratio 6:1). Later, 2-D packed bed and channel models were developed $[1,4,50]$. For thick seams with low-pressure operations (< $1 \mathrm{MPa}$ ), a CAVSIM process was developed [48]. Progress in computing has enabled the development of 3-D models of UCG, e.g., a 3-D computational model for roof spalling, bed dynamics, and cavity growth were developed in [5]. An important aspect of UCG models are the assumptions and modeling of the chemical reactions in the process. Models based on a chemical reaction of UCG can be found in $[8,47]$. The comprehensive summary of reported UCG models can be found in $[27,45]$.

A special group consists of mathematical models based on soft sensors where an unmeasurable process variable is calculated or estimated from other measurable process variables. Since the UCG runs in the underground, some of the process variables cannot be measured directly by conventional hardware (e.g., underground temperature). Known proxies of underground temperature estimation are based on measurement of carbon isotopes [11,28] and radon emanations $[54,58]$. Various soft-sensing methods are developed for monitoring and prediction of UCG variables. Currently, UCG modeling with the application of machine learning (i.e., neural networks (NNs), support vector machines (SVMs), and adaptive regression models) is developed. This approach brings interesting results in the modeling of expert knowledge of the UCG. It is mostly data-driven modeling with continuous model adaptation as the process evolves. In terms of control, it needs to predict the composition of syngas calorific value, or underground temperature in a combination of control variables.

Some works model underground temperature based on nonstationary heat conduction (e.g., [17, 29]). Recently, many models based on neural networks were developed (e.g., [23, 26, 35, 36]). Some models are directly developed to support predictive control. Various applications of support vector machines (SVMs) for UCG data prediction can be found in the literature. For example, Kačur et al. [25] achieved interesting results in predicting calorific value using support vector regression (SVR). Other methods of prediction applied in UCG that utilize soft computing can be found in [22, 37, 60, 61].

\subsection{Control of UCG}

The control of UCG to produce stable syngas is most problematic. Currently, the UCG is controlled blindly, causing unstable quality of syngas. For air technology (i.e., the air is used as a main gasification agent), a lower syngas calorific value of $3-7 \mathrm{MJ} / \mathrm{Nm}^{3}$ is achieved. In oxygen technology (i.e., typically a mixture of oxygen and water vapor), the calorific value is usually in the range of 9-14 MJ/ $\mathrm{Nm}^{3}$ [39]. Optimal control is the most investigated field in UCG within academic research. Much work focused on UCG control has been done on experimental ex-situ reactors (e.g., [24, 30, 31]) but also there are some trials on in-situ gasifier (e.g., [52]).

The discrete PI controller is ideal for slow and time-delayed processes such as UCG. Applications of PI controllers to stabilize the oxygen in syngas and underground temperature can be found in [24]. Kačur and Kostúr proposed PI controllers using discrete ARX models and a modified Ziegler-Nichols method [24]. The authors also verified the PI controller with a continuous adaptation of controller parameters. By such an approach, they were able to increase and maintain the syngas calorific value. Another approach to automated UCG control was based on the application of the adaptive regression model [33]. The proposed regression model was able to calculate optimal manipulation variables (i.e., volume flows of gasification agents) with the continual adaptation of model parameters from the historical data. Recently, Kostúr and Kačur [30] developed optimal control based on the utilization of the gradient optimization method. This method was adopted to continually optimize manipulation variables (i.e., gasification agents, and outlet underpressure) and maximize syngas calorific value during UCG. It was a model-free control of UCG verified in laboratory conditions on ex-situ reactor. Kostúr and Kačur [31] also proposed and verified an extremum-seeking control to maximize $\mathrm{CO}$ in syngas using air as one manipulation variable.

Uppal et al. [52,53] have also done a lot of research in UCG control. They have proposed a predictive model of the one-dimensional underground coal bed. Designed model-based control optimizes UCG inputs. The model has been connected in a closed loop with a robust slide mode controller (SMC) to stabilize the 
calorific value of syngas to the reference value. Other researchers, i.e., Wei and Liu [55], proposed a UCG control scheme based on iterative optimal learning and iterative adaptive dynamic programming. Some connection of learning methods with predictive control of gasification can be found in [20]. In this work, adaptive predictive control of oxygen concentration in syngas without the use of a model was proposed. The authors used offline input-output data for the learning method and compact-form dynamic plant linearization.

\subsection{Potential of Predictive Control in UCG}

A model predictive control (MPC) represents a robust control technique that can be applied on multivariable linear and nonlinear processes. Since the coal gasifier appears to be a non-linear system with some inputs and outputs, this way of managing it can have potential benefits. The main objective is to continuously maintain the syngas calorific value on desired value over time as the UCG develops (e.g., combustion front propagation, water influx, gas leaks, and various uncertainties). Unfortunately, there is no model to describe UCG comprehensively, but different black-box models have potential. The technique of MPC integrates optimal control, stochastic control, and control of processes with dead time. In this control technique, the model of process and minimizing of the cost function is used to obtain optimal control. There are many applications of MPC in the gasification industry (e.g., [3, 7, 59, 62]). Unfortunately, only low evidence of this technique can be found in UCG. Beneficial is MPC formulation in the state space [43]. It facilitates the generalization of MPC for mono-variable, multi-variable, linear, and nonlinear processes. When control multiple-inputs-multiple-outputs (MIMO) or multiple inputs-single-outputs (MISO), the most popular way is decoupling MISO control into multiple single-input-single-output (SISO) controllers (e.g., PID control). However, complete decoupling is very difficult to achieve for processes with complex dynamics or dead times. In MPC, the MISO and MIMO systems are controlled in a straightforward manner [13, 15, 46].

Although many industrial processes are nonlinear, most MPC applications are based on the use of linear models. In most cases, the nonlinear model of the process can be linearized in operating points where linearity is assumed in the neighborhood of a specific operating point. The linear model can be relative easy identified on process data and provide a good result when the plant is operating in the neighborhood of the operating point. In this paper, a model-based MARS is linearized to the discrete state-space model in the operating point by offline and appropriately used within the linear MPC strategy. The online model estimation and continual MPC model update ensure the adaptation of MPC. MARS is an attractive tool to model nonlinear processes. This feature, together with training availability, makes it very useful in MPC applications to process imitation. The similar utilization can be found in NNs or SVMs. This paper proposes adaptive model predictive control (AMPC) using online model estimation and applies it to the MARS model of UCG that imitates the real process.

\section{Physical Modeling of UCG}

Researchers have developed various ex-situ gasification plants to improve the UCG. Ex-situ gasification was most investigated in $[16,31,32,56]$. To investigate the possibilities of control and measurement of process variables in the UCG, an ex-situ reactor has been created.

Two compressors injected air into the pressure vessel from where it was blown into the ex-situ reactor. Technical oxygen as the second gasification agent was added from pressure vessels. Air and oxygen were mixed in the mixing chamber, and this mixture was injected into the ex-situ reactor to support the gasification. The flow rates of gasification agents were controlled using valves.

At the outlet, the composition of syngas was measured by stationary analyzers. The calorific value of syngas was calculated from the measured syngas composition. The underpressure at the outlet was controlled by an industrial fan and measured by a pressure gauge. The physical model of the underground coal bed was formed by blocks of Lignite-type coal from the Slovak mine (see Figure 2). Thermocouples of K-type were used to measure temperature in coal, overburden, underburden and gasification channels. To meet similarity conditions, the reactor was slightly inclined and sealed. Several experiments were performed on two ex-situ reactors (i.e., syngas generators 1 and 2). The 
Figure 2

Creation of the physical model of the coal seam

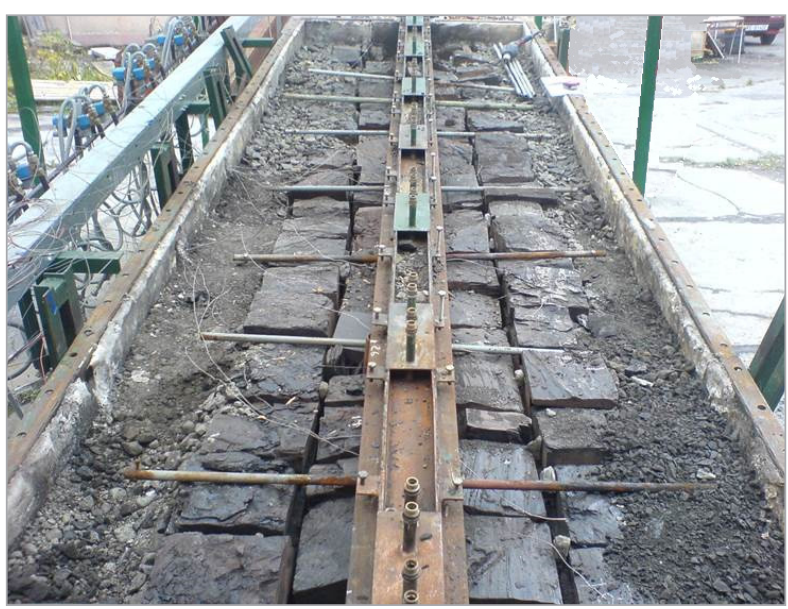

comprehensive scheme of the experimental gasification equipment with two syngas generators is shown in Figure 3. These ex-situ reactors differ in dimensions. The bedding of coal corresponds to real coal seam with inclination. A monitoring system to monitor experiments was created in the SCADA/HMI application Promotic (see Figures 4 and 5). The monitoring system provided auxiliary algorithms for op- erating of the control system, plotting and archiving measured data from the gasification process to the database. The data recorded from experimental gasification during laboratory gasification were also used in this paper to verify the proposed modeling method. The monitoring system was divided into screens, between which the operator may switch.

The control of experimental UCG was ensured by PLC (B\&R X20) that performed several cyclic tasks (i.e., data acquisition, data processing, process variables stabilization via PI controllers, extremum seeking control, and optimal control based on gradient method). The PLC was connected with PC through RS232 and OPC protocol.

The proposed monitoring system for experimental gasification equipment is able to monitor and record various process variables, e.g., injected air volume flow, injected air overpressure, injected oxygen volume flow, injected oxygen overpressure, exhausting underpressure, syngas temperature, concentrations of $\mathrm{O}_{2}, \mathrm{CO}_{2}, \mathrm{H}_{2}, \mathrm{CO}, \mathrm{CH}_{4}$ in syngas, calorific value of syngas, syngas volume flow, temperatures of overburden layers, temperatures inside gasifier (i.e., in oxidizing, reducing, and pyrolyzing zone), and temperatures of underburden layers.

\section{Figure 3}

Experimental ex-situ reactors with devices for measurement and control.

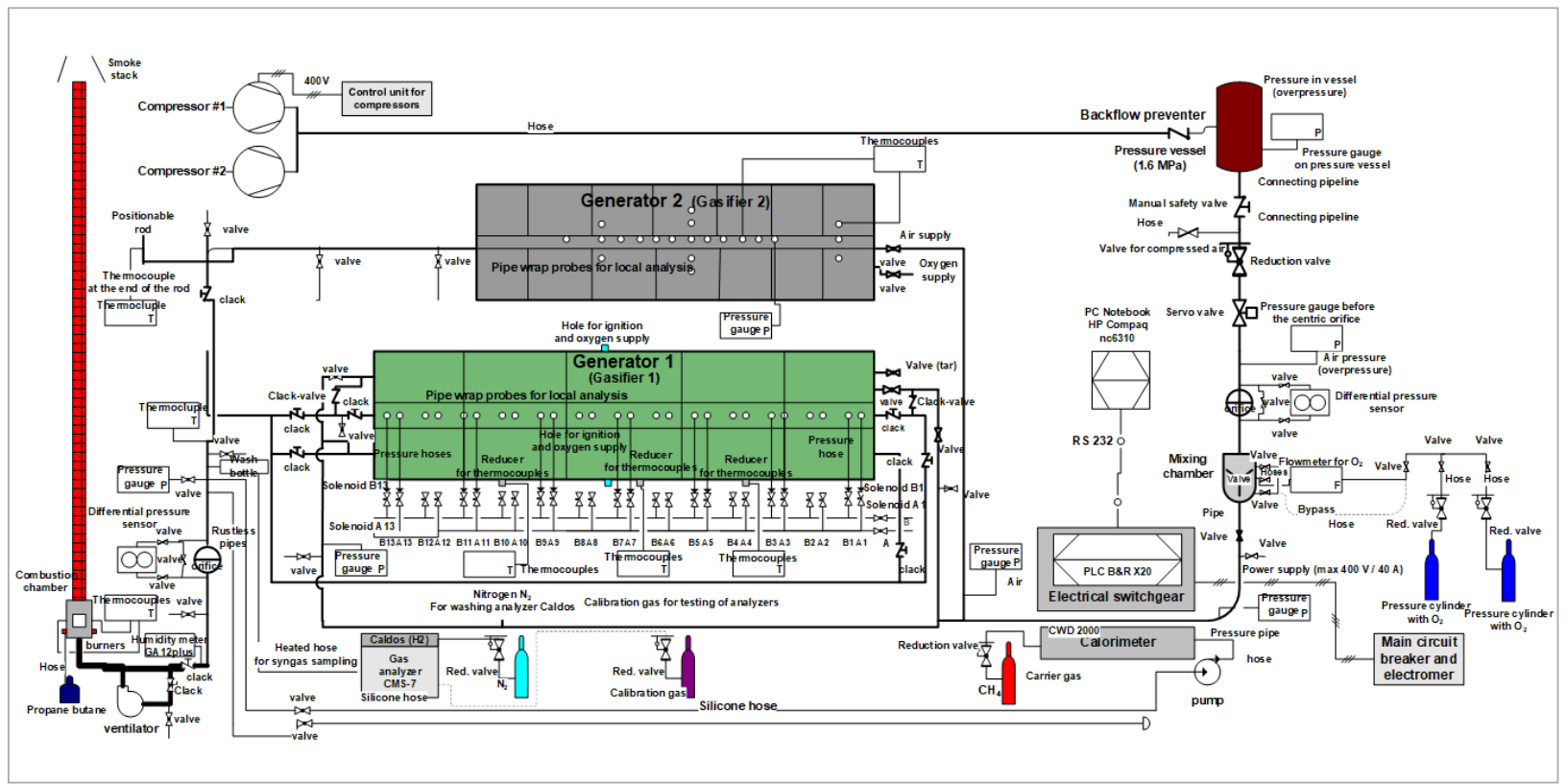




\section{Figure 4}

The main screen of the monitoring system

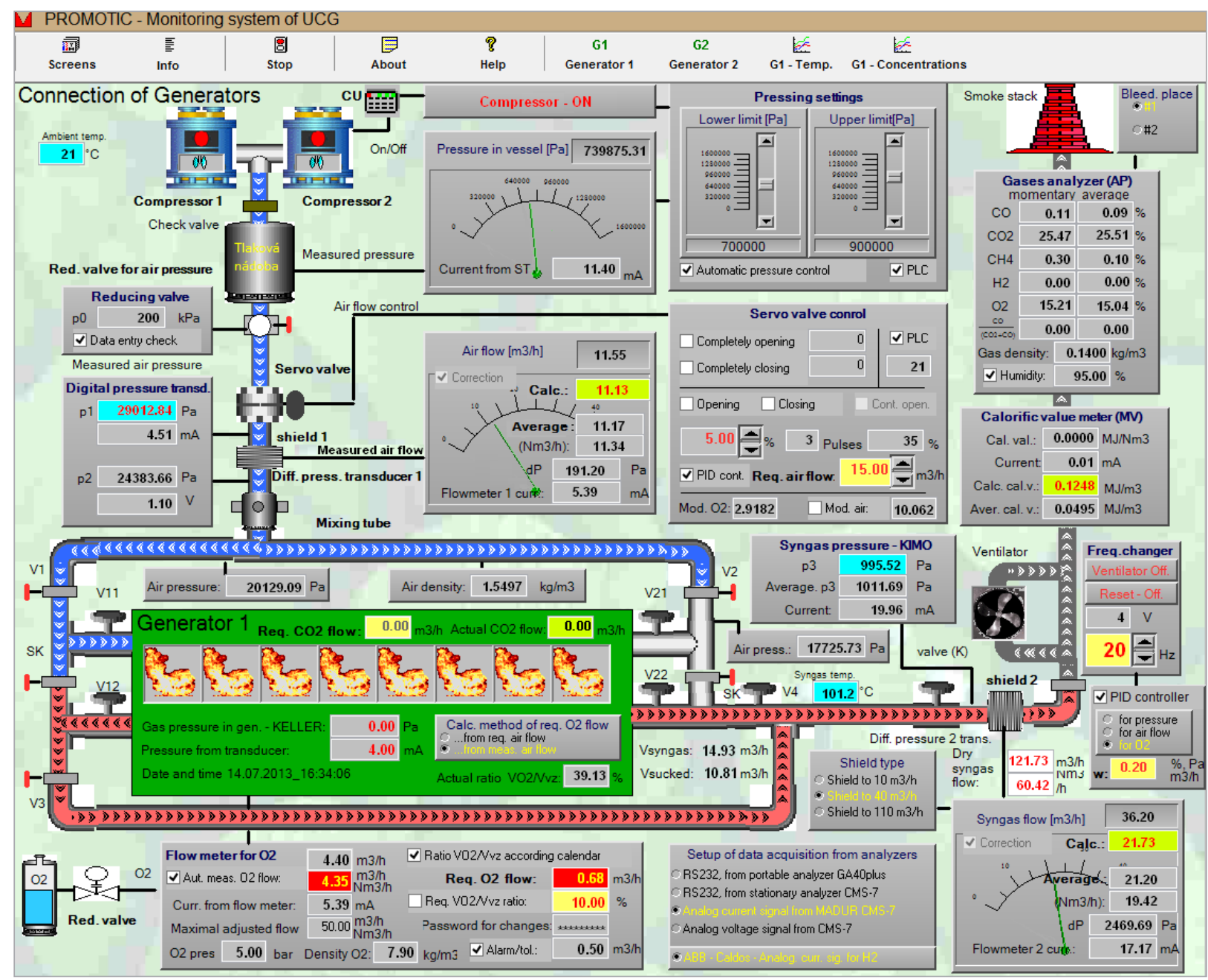

\section{Figure 5}

(a) Monitoring temperatures in the physical model, (b) Trends of various process variables

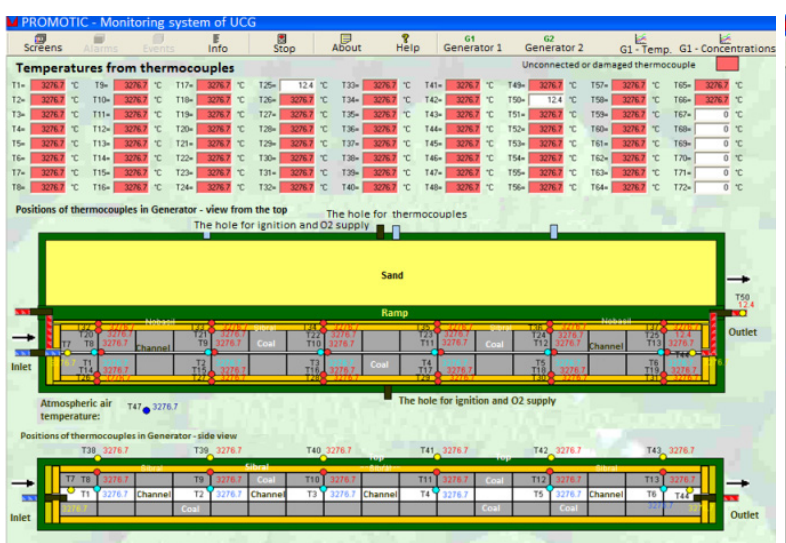

(a)

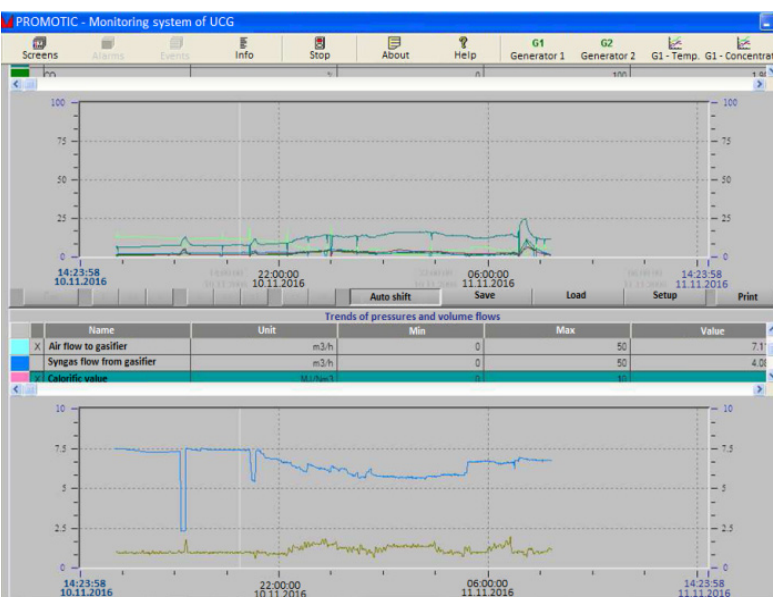

(b) 


\section{UCG Modeling Based on MARS}

Currently, various methods for nonlinear system modeling are applied in research, e.g., Neural Networks (NN), Nonlinear Finite Impulse Response (NFIR), Nonlinear AutoRegressive with eXogenous inputs (NARX), Nonlinear AutoRegressive Moving Average with eXogenous input (NARMAX), Nonlinear Output Error (NOE) model, and Nonlinear Box-Jenkins (NBJ) model. This section explains the background of the black-box method called Multivariate Adaptive Regression Splines (MARS) that can model nonlinearities in the UCG process. The model can be created from offline UCG observations and targets. The goal is to find the dependency of variables $\mathrm{y}_{i}$ on one or more independent variables $\mathbf{u}_{i}$. The following regression sample is considered:

$$
D=\left\{\mathbf{u}_{i}, y_{i}\right\}_{i=1}^{N}=\left\{u_{1 i}, \ldots, u_{n i}, y_{i}\right\}_{i=1}^{N},
$$

where $\mathbf{u}_{i} \in \mathbb{R}^{n}$ represents the $i$-th vector of the independent variables; $y_{i} \in \mathbb{R},(i=1, \ldots, N)$ is the dependent variable, i.e., target; $n$ represents the number of independent variables, and $N$ represents the number of samples, i.e., the total number of $\left(\mathbf{u}_{i}, y_{i}\right)$ pairs. These variables represent manipulated variables and measured output in UCG. In experimental UCG, three manipulation variables (i.e., input observations) and two controlled variables (i.e., targets) have been specified (see Table 1).

Table 1

Selected observations and targets in UCG

\begin{tabular}{l|l}
\multicolumn{1}{c|}{ Observations } & \multicolumn{1}{c}{ Targets } \\
\hline \begin{tabular}{l|}
$u_{1}-$ Air flow \\
$\left(\mathrm{Nm}^{3} / \mathrm{h}\right)$
\end{tabular} & \\
\hline$u_{2}-$ Oxygen flow & $y_{1}-$ Syngas calorific \\
$\left(\mathrm{Nm}^{3} / \mathrm{h}\right)$ & value $\left(\mathrm{MJ} / \mathrm{Nm}^{3}\right)$ \\
\cline { 1 - 1 }$u_{3}-$ Outlet relative pressure $(\mathrm{Pa})$ & $y_{2}-$ Underground \\
& temperature $\left({ }^{\circ} \mathrm{C}\right)$ \\
\hline
\end{tabular}

The MARS method was firstly introduced in [18] and represents a non-parametric regression technique that can automatically model nonlinearities and interactions between process variables. If the MARS technique of regression analysis on time series is used, the autoregressive model can be obtained. Many works have been published that discussed the MARS method (e.g., [14, 19, 51]).
In order to create the MARS model, the training data vectors, i.e., the inputs (observations) and outputs (targets) are needed. Training data are splitinto several splines on an equivalent interval basis. The data are in every spline split into many subgroups, and several knots are created that can be placed between different input variables or different intervals in the same input variable to separating subgroups [14]. To verify the performance of the model being created, the model is verified on the test data. In MARS, the regression function called basis function (BF) is approximated by smoothing splines for a general representation of data in each subgroup. Between any two knots, the model can characterize the data either globally or by using linear regression. The $\mathrm{BF}$ is unique between any two knots and is shifted to another BF at each knot. Two BFs in two adjacent domains of data intersect at the knot to make model outputs continuous. MARS creates a curved regression line to fit the data from subgroup to subgroup and from one spline to another spline. For evading over-fitting and over-regressing, the shortest distance between two neighboring knots is predetermined to prevent too few data in a subgroup [18]. In the MARS method, the goal is to find the dependency of variables $y_{i}$ on one or more independent variables $\mathbf{u}_{i}$. The relationship between $y_{i}$ and $\mathbf{u}_{i}(i=1, \ldots, N)$ can be represented as:

$$
y_{i}=f\left(u_{i}^{1}, u_{i}^{2}, \ldots, u_{i}^{n}\right)+\varepsilon=f\left(\mathbf{u}_{i}\right)+\varepsilon,
$$

where $f$ is an unknown function, and $\varepsilon$ is an error $\left(\varepsilon \sim N\left(0, \sigma^{2}\right)\right)$. The single-valued deterministic function $f$ captures the joint predictive relationship of $y_{i}$ on $\left(u_{i}^{1}, u_{i}^{2}, \ldots, u_{i}^{n}\right)$. The additive stochastic component $\varepsilon$ usually reflects the dependence of $y_{i}$ on values other than $\left(u_{i}^{1}, u_{i}^{2}, \ldots, u_{i}^{n}\right)$, that are neither controlled nor observed.

In the one-dimensional case, splines are expressed in terms of piecewise linear basis functions, $(u-t)$ and $(t-u)_{+}$with the node in $t$. The "+" means a positive part. These functions are truncated linear functions, for $u \in \mathbb{R}$ :

$$
\begin{aligned}
(u-t)_{+} & =\left\{\begin{array}{l}
u-t, \quad \text { If } u>t, \\
0, \quad \text { otherwise }
\end{array}\right. \text { and } \\
(t-u)_{+} & =\left\{\begin{array}{l}
t-u, \quad \text { If } u<t, \\
0, \quad \text { otherwise. }
\end{array}\right.
\end{aligned}
$$

Each function (i.e., $(u-t)_{+}$and $\left.(t-u)_{+}\right)$is piecewise linear, with a knot at the value $t$. They are marked as 
linear splines. These two functions are named as a reflected pair. In the multidimensional case, the idea is to form reflected pairs for each input component $u^{j}$ of the vector $\mathbf{u}=\left(u^{1}, \ldots, u^{j}, \ldots, u^{n}\right)^{T}$ with knots at each observed value $u_{i}^{j}$ of that input $(i=1,2, \ldots, N ; j=1,2, \ldots, n)$. Thus, a set of constructed basis functions can be expressed as follows:

$$
\begin{aligned}
C= & \left\{\left(u^{j}-t\right)_{+},\left(t-u^{j}\right)_{+} \mid t \in\left\{u_{1}^{j}, u_{2}^{j}, \ldots, u_{N}^{j}\right\},\right. \\
& j \in\{1,2, \ldots, n\}\} .
\end{aligned}
$$

If all input data are different, then in the set of $2 N n$ basis functions, each of them depends on only one variable $u^{j}$. For example, $B(\mathbf{u})=\left(u^{j}-t\right)_{+}$is regarded as a function over the entire input space $\mathbb{R}^{n}$. Basis functions used for approximation are as follows:

$$
B_{m}(\mathbf{u})=\prod_{k=1}^{K_{m}}\left[s_{k, m} \cdot\left(u^{v(k, m)}-t^{k m}\right)\right]_{+},
$$

where $K_{m}$ represents the total number of truncated linear functions in the $m$-th basis function, $u^{(v(k, m))}$ is the component of the vector $\mathbf{u}$, related to the $k$-th truncated linear function in the $m$-th basic function, $t^{k m}$ is the corresponding node, and $s_{k, m} \in\{ \pm 1\}$. Parameter $K_{m}$ is by user-defined degree order of the interaction term and $s_{k, m}$ represents the direction of the univariate term, which could be positive or negative.

The model-building strategy is like a forward stepwise linear regression, but instead of using the original inputs, it is allowed to use functions from the set $C$ of their products. Therefore, the MARS model can be expressed by the following equation

$$
y=\hat{f}(\mathbf{u})+\varepsilon=c_{0}+\sum_{m=1}^{M} c_{m} B_{m}(\mathbf{u})+\varepsilon,
$$

where $y$ is the output variable, $\mathbf{u}$ represents the vector of input variables, $M$ is the number of basis functions in the model (i.e., number of spline functions), $c_{0}$ is the coefficient of the constant basis function $B_{0}$, and the sum is over the basis functions $B_{m}$ produced by an algorithm that implements the forward stepwise part of the MARS strategy by incorporating the modification to recursive partitioning. The coefficients $c_{m}$ are estimated by minimizing the residual sum-of-squares. $B_{m}(\mathbf{u})$ is the $m$-th function in $C$, or a product of two or more such functions.
The most important thing in this model is the choice of the basis functions. In the beginning, the model contains a single function $B_{0}(\mathbf{u})=1$ and all functions from the set $C$ are possible candidates for inclusion in the model. As in linear regression, setting $B_{m}$, the coefficients $c_{m}$ can be found by the method of least squares. Another subroutine of MARS performs the backward deletion strategy wherein each iteration causes one unnecessary (i.e., redundant) basis function to be deleted. A function whose removal either mostly improves the fit or at least degrades it will be deleted. However, the constant basis function $B_{1}(\mathbf{u})=1$ is never removed.

\section{Model Predictive Control}

Nowadays, various methods of technological process control are being developed, e.g., [42, 44]. The MPC controller is conceived as multidimensional, i.e., it works in a coordinated manner with a higher number of manipulated variables (MVs) and controlled variables, i.e., measured outputs (MOs) what is typical in MIMO or MISO systems. All limitations on permitted ranges of values and rate of change of regulated and action variables are already respected when calculating action interventions and not additionally. At any time of sampling, the following must be available: dynamic control system model, including restrictive conditions; current and past values of controlled variables; previous values of manipulated variables; known or expected course of desired values of controlled variables within the assumed horizon of prediction $N$. For comparison, MPC responds to the predicted future value of control errors, PID only to current and past values. At each moment of sampling, the optimization task is solved, and the calculation of manipulated variables is performed within the considered control horizon $N_{u}$. The control sequence has the following form:

$$
\begin{aligned}
& \mathbf{u}(k \mid k), \mathbf{u}(k+1 \mid k), \mathbf{u}(k+2 \mid k), \\
& \ldots, \mathbf{u}\left(k+N_{u}-1 \mid k\right) .
\end{aligned}
$$

It is assumed that $\mathbf{u}(k+p \mid k)=\mathbf{u}\left(k+N_{u}-1 \mid k\right)$ for $p \geq N_{u}$. The calculation of the manipulated variable is based on minimizing the difference between the predicted values of the controlled variable and the future course 
of the vector of desired values $\mathbf{w}(k+p \mid k)$ within prediction horizon $N(p=1,2, \ldots, N)$ (see Figure 6). Only $\mathbf{u}(k \mid k)$ is used in the calculation sequence, and the calculation is repeated in the next step. Horizon is moving so gradually. This procedure is referred to as a receding horizon. The MPC control calculations based on actual measurements and predictions are carried at individual sampling periods $T_{s}$. These calculations include process constraints and other parameters that can be manually specified. The main task of the MPC controller is to determine a sequence of control moves in the manipulated variable so that the system can be tracked to its setpoint in an optimal fashion. Usually, the prediction horizon $N$ and control horizon $N_{u}$ are set so that $N_{u} \leq N$ which results in that the MPC to be less sensitive to model [2].

\section{Figure 6}

Principle of MPC

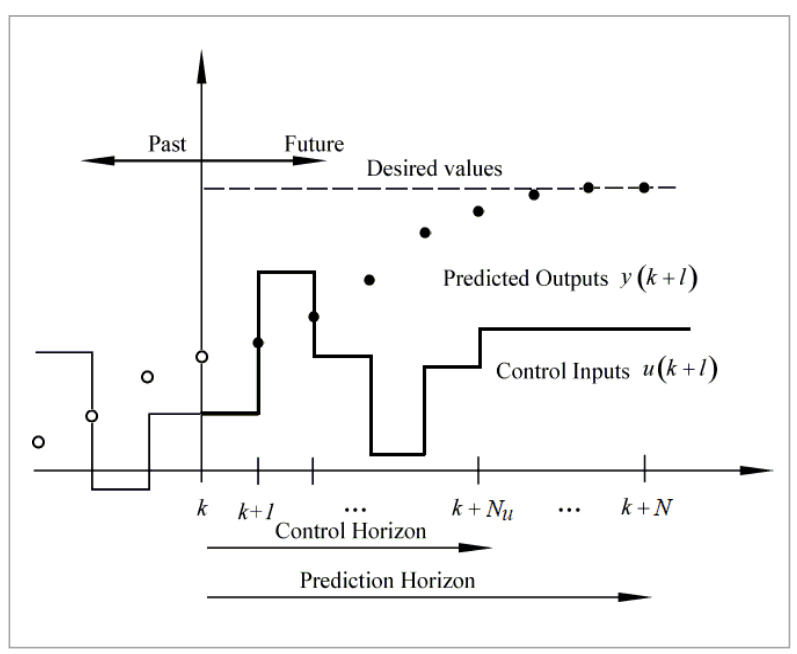

The future plant outputs calculated via predictive model i.e., $\mathbf{y}(k+p)$, for $p=1,2, \ldots, N$, (where $N$ is the prediction horizon) depend on plant current states $\mathbf{x}(\mathrm{k})$ and future values of manipulating variables (MVs), i.e., $\mathbf{u}(k+p)$, for $p=1,2, \ldots, N_{u}$ (where $N_{u}$ is the control horizon and $\left.N_{u} \leq N\right)$.

\subsection{State-Space Interpretation of MPC}

In industry, state-space models based on step response [34, 38] are widely used. If a MIMO system (i.e., multivariable process) without disturbance is considered, the Equation (8) represents the general form of the state-space model:

$$
\mathbf{x}(k+1)=\mathbf{A} \mathbf{x}+\mathbf{B}_{u} \mathbf{u}(k),
$$$$
\mathbf{y}(k)=\mathbf{C} \mathbf{x}(k) \text {. }
$$

The general prediction state-space model (i.e., the internal model of MPC) that assumes input disturbance (e.g., in the form of unit gains) represents Equations (9):

$$
\begin{aligned}
& \mathbf{x}(k+1)=\mathbf{A} \mathbf{x}(k)+\mathbf{B}_{u} \mathbf{u}(k)+\mathbf{B}_{v} \mathbf{v}(k)+\mathbf{B}_{d} \mathbf{d}(k), \\
& \mathbf{y}(k)=\mathbf{C} \mathbf{x}(k)+\mathbf{D}_{\boldsymbol{v}} \mathbf{v}(k)+\mathbf{D}_{d} \mathbf{n}_{d}(k) .
\end{aligned}
$$

These equations represent linear discrete-time, statespace plant model where the matrices $\mathbf{A}, \mathbf{B}_{w}, \mathbf{B}_{v}, \mathbf{B}_{d}, \mathbf{C}$, $\mathbf{D}_{v}$ and $\mathbf{D}_{d}$ can vary with time. Parameter $k$ is the time index (i.e., current control interval ); $\mathbf{x}$ represents the state vector of $n_{x}$ plant model states; $\mathbf{u}$ is the vector of $n_{u}$ manipulated variables (MVs), which are adjusted by the MPC controller; $\mathrm{v}$ represents the vector of $n_{v}$ measured disturbance inputs (if any); $\boldsymbol{d}$ is the vector of $\mathbf{n}_{d}$ unmeasured disturbance inputs and $\mathbf{y}$ is the vector of $n_{y}$ plant outputs. If $\Delta \mathbf{u}(k)=\mathbf{u}(k)-u(k-1)$ is used instead $\mathbf{u}(k)$, then an incremental state-space form of the model can be obtained. Equation (10) shows the future plant behavior estimated by the model at time step $k=0$ when setting $\mathbf{n}_{d}(i)=0$ for all prediction steps $i[6]$ :

$$
\begin{aligned}
& \boldsymbol{y}(i \mid 0)= \\
& \mathbf{C}\left[\mathbf{A}^{i} \mathbf{x}(0)+\sum_{h=0}^{i-1} \mathbf{A}^{i-1}\left(\mathbf{B}_{u}\left(\mathbf{u}(-1)+\sum_{j=0}^{h} \mathbf{\Delta} \mathbf{u}(j)\right)+\mathbf{B}_{v} v(h)\right)\right]+\mathbf{D}_{v} \mathbf{v}(i) .
\end{aligned}
$$

This equation gives the solution

$$
\left[\begin{array}{c}
\mathbf{y}(1) \\
\ldots \\
\mathbf{y}(p)
\end{array}\right]=\mathbf{S}_{x} \mathbf{x}(0)+\mathbf{S}_{u \mathbf{u}} \mathbf{u}(-1)+\mathbf{S}_{u}\left[\begin{array}{c}
\Delta \mathbf{u}(0) \\
\ldots \\
\Delta \mathbf{u}(p-1)
\end{array}\right]+\mathbf{H}_{v}\left[\begin{array}{c}
\mathbf{v}(0) \\
\ldots \\
\mathbf{v}(p)
\end{array}\right],
$$

where

$$
\mathbf{s}_{\boldsymbol{x}}=\left[\begin{array}{c}
\mathbf{C A} \\
\mathbf{C A}^{2} \\
\cdots \\
\mathbf{C A}^{p}
\end{array}\right] \in \mathrm{R}^{p n_{y} \times n_{x}},
$$




$$
\begin{aligned}
\mathbf{S}_{u 1} & =\left[\begin{array}{c}
\mathbf{C B}_{u} \\
\mathbf{C} \mathbf{B}_{u}+\mathbf{C A} \mathbf{B}_{u} \\
\cdots \\
\sum_{h=0}^{p-1} \mathbf{C A}^{h} \mathbf{B}_{u}
\end{array}\right] \in \mathrm{R}^{p n_{y} \times n_{u}}, \\
\mathbf{S}_{u} & =\left[\begin{array}{cccc}
\mathbf{C B}_{u} & 0 & \cdots & 0 \\
\mathbf{C A B}_{u} & \mathbf{C B}_{u} & \cdots & 0 \\
\cdots & \cdots & \cdots & \cdots \\
\sum_{h=0}^{p-1} \mathbf{C A}^{h} B_{u} & \sum_{h=0}^{p-2} \mathbf{C A}^{h} \mathbf{B}_{u} & \cdots & \mathbf{C B}_{u}
\end{array}\right] \in \mathrm{R}^{p n_{y} \times n_{u}}, \\
\mathbf{H}_{v} & =\left[\begin{array}{ccccc}
\mathbf{C B}_{v} & \mathbf{D}_{v} & 0 & \cdots & 0 \\
\mathbf{C A B}_{v} & \mathbf{C B}_{v} & \mathbf{D}_{v} & \cdots & 0 \\
\cdots & \cdots & \cdots & \cdots & \cdots \\
\mathbf{C A}^{p-1} \boldsymbol{B}_{V} & \mathbf{C A}^{p-2} \mathbf{B}_{v} & \mathbf{C A}^{p-3} \mathbf{B}_{v} & \cdots & \mathbf{D}_{v}
\end{array}\right] \in \mathrm{R}^{p n_{y} \times(p+1) n_{v}} .
\end{aligned}
$$

When control law depends on states $\mathbf{x}(k)$, then an appropriate state observer is needed to be used [6].

In MPC, the optimization problem is solved by quadratic programming (QP) to determine optimal manipulated variables (MV) in each control interval. Constraints represent conditions that must be satisfied (i.e., physical bounds on MVs and plant outputs). In MPC, a decision $\mathbf{z}_{k}$ about MVs adjustment must be obtained by minimizing the cost function (13) in each control interval [6]:

$$
\begin{aligned}
J\left(\mathbf{z}_{\boldsymbol{k}}\right)=\sum_{i=1}^{p-1}\left\{\left[\mathbf{e}_{\boldsymbol{y}}^{T}(k+i) \mathbf{Q} \mathbf{e}_{\boldsymbol{y}}(k+i)\right]+\right. \\
{\left[\mathbf{e}_{u}^{T}(k+i) \mathbf{R}_{u} \mathbf{e}_{u}(k+1)\right]+} \\
{\left[\Delta \mathbf{u}^{T}(k+i) \mathbf{R}_{\Delta u} \Delta \mathbf{u}(k+i]\right\}+\rho_{\varepsilon} \varepsilon_{k}^{2}, }
\end{aligned}
$$

where $\mathbf{Q}\left(n_{y} \times n_{y}\right), \mathbf{R}_{w}$, and $\mathbf{R}_{\Delta u}\left(n_{u} \times n_{u}\right)$ are positive-semi-definite weight matrices. These matrices are diagonal with the squares of those weights as diagonal elements. $\mathbf{e}_{y}(k+i)=\mathbf{S}_{y}{ }^{-1}[\mathbf{r}(k+i+1 \mid k)-\mathbf{y}(k+i+1 \mid k)]$; $\mathbf{e}_{u}(i+k)=\mathbf{S}_{u}{ }^{-1}\left[\mathbf{u}_{\text {target }}(k+i \mid k)-\mathbf{u}(k+\mathrm{i} \mid k)\right] ; \quad \Delta \mathbf{u}(k+\mathrm{i})=\mathbf{S}_{u}{ }^{-1}[\mathbf{u}$ $(k+i \mid k)-\mathbf{u}(k+i-1)] ; k$ is the current control interval, $p$ is the prediction horizon (i.e., number of intervals), $n_{u}$ represents the number of MVs, $n_{y}$ represents the number of the measured output variables, $\mathbf{S}_{y}$ is a diagonal matrix of the plant output variable sale factors, $\mathbf{S}_{u}$ is a diagonal matrix of MV scale factors. $\mathbf{r}(k+1 \mid k)$ represents the vector of $n_{y}$ plant output reference values at the $i$-th prediction horizon step. $\mathbf{y}(k+1 \mid k)$ represents $n_{y}$ measured plant outputs at the $i$-th prediction horizon step. Vector $\mathbf{z}_{k}$ represents the QP decision, given by:

$\mathbf{z}_{k}^{T}=\left[\mathbf{u}(k \mid k)^{T} \mathbf{u}(k+1 \mid k)^{T} \ldots \mathbf{u}(k+p-1 \mid k)^{T} \varepsilon_{k}\right]$,

where $\mathbf{u}_{\text {target }}(k+i \mid k)$ are $n_{u}$ MV target values corresponding to $\mathbf{u}(k+\mathrm{i} \mid k)$. Parameter $\varepsilon_{k}$ is scalar QP slack variable at control interval $k$ (dimensionless) used for constraint softening and $\rho_{\varepsilon}$ is constraint violation penalty weight (dimensionless).

When using MPC, an optimization problem is solved at each time step $k$ through the cost function based on output predictions over a prediction horizon of $p$ time steps; this objective function (usually a quadratic one) is minimized by a selection of manipulated variables moves over a control horizon of $M$ control moves. It is important to emphasize that, even though at each time step a group of $M$ moves is calculated, only the first one $\mathbf{u}(k)$ is implemented. After this step, the measurement at the next time instant $\mathbf{y}(k+1)$ is obtained, followed by a correction due to model error, and then a new optimization problem is solved again. These procedures are carried for every time step $k$.

In MPC applied on MIMO system, the following form of constraints can be considered [6]:

$$
\begin{aligned}
& \frac{y_{j, \text { min }}}{s_{j}^{y}}-\varepsilon_{k} V_{j, \text { min }}^{y}(i) \leq \frac{y_{j}(k+i \mid k)}{s_{j}^{y}} \leq \frac{y_{j, \text { max }}(i)}{s_{j}^{y}}+\varepsilon_{k} V_{j, \text { max }}^{y}(i), \\
& \left(i=1, \ldots, p ; j=1, \ldots, n_{y}\right), \\
& \frac{u_{j, \text { min }}}{s_{j}^{u}}-\varepsilon_{k} V_{j, \text { min }}^{u}(i) \leq \frac{u_{j}(k+i-1 \mid k)}{s_{j}^{u}} \leq \frac{u_{j, \text { max }}(i)}{s_{j}^{u}}+\varepsilon_{k} V_{j, \text { max }}^{u}(i), \\
& \left(i=1, . ., p ; j=1, \ldots, n_{y}\right), \\
& \frac{\Delta u_{j, \text { min }}}{s_{j}^{u}}-\varepsilon_{k} V_{j, \text { min }}^{\Delta u}(i) \leq \frac{\Delta u_{j}(k+i-1 \mid k)}{s_{j}^{u}} \leq \frac{\Delta u_{j, \text { max }}(i)}{s_{j}^{u}}+\varepsilon_{k} V_{j, \text { max }}^{\Delta u}(i), \\
& \left(i=1, \ldots, p ; j=1, \ldots, n_{y}\right),
\end{aligned}
$$

where parameters $V_{j, \text { min }}{ }^{y}, V_{j, \text { max }}{ }^{y}, V_{j, \text { min }}{ }^{u}, V_{j, \text { max }}{ }^{u}, V_{j, \text { min }}{ }^{\Delta u}$, and $V_{\text {jmax }} \Delta u$ are dimensionless controller constants analogous to the cost weights used for constraint softening; $\varepsilon_{k} \geq 0$ is the scalar QP slack variable used for constraints softening; $s_{j}^{y}$ is the scale factor for the $j$-th plant out- 
put; $y_{j, \text { min }}$ and $\mathrm{y}_{j, \text { max }}(i)$ are lower and upper limits for the $j$-th plant output and the $i$-th prediction horizon step; $u_{j \text { min }}$ and $u_{j, \text { max }}(i)$ are lower and upper limits for the $j$-th MV and the $i$-th prediction horizon step; $\Delta u_{j, m i n}$ and $\Delta u_{j, \text { max }}(i)$ are lower and upper limits for the $j$-th MV increment and the $i$-th prediction horizon step.

\section{Results and Discussion}

\subsection{Modeling Results}

This paper simulates in-situ UCG plant relying on an ex-situ physical model (see Section 2) and UCG process by the MARS method. An open MARS source code (i.e., ARESLab software) [21] was used to create the model and the necessary simulations. The UCG experiment was performed on a generator 1 , and measured data were recorded in a database to create a regression model. The obtained process model can imitate the nonlinear behavior of UCG. The initial number of BFs in the forward phase was determined according to the formula: $\min (200, \max (20,2 d))+1$, where $d$ represents the number of input variables. The initial number of BFs was set to 21 in all simulations. There was regarded maximal interactivity between the input variables and no self-interactions any input variable. The piecewise-linear type of the model has been analyzed in order to know the prediction performance in two and three input variables. The best or optimal number of maximal BFs in final MARS model was estimated by the Generalized Cross-Validation criterion. Piecewise linear type of MARS model uses $\max (0, u-t)$ function, where $t$ is the knot. The max function represents the positive part of $(0, u-t)$ what can be formally expressed as the following:

$$
\max (0, u-t)=\left\{\begin{array}{l}
u-t, \quad \text { if } u \geq t \\
0,
\end{array}\right.
$$

where $t$ represents a univariate knot which is selected for each of the factor variables $u$.

The first stage of the proposed MPC is to train a MARS model to represent the forward dynamics of the plant. The MARS model uses an offline dataset of input observations (MVs) and targets for the training itself. When specific MVs inputs into the model, the predicted value at given inputs can be obtained. The offline data should be collected from the real UCG operation but in our case, the data from experimental UCG on ex-situ reactor have been used. For best model approximation, the database should be continuously updated with newer input-output pairs and repeat learning. Although the MARS prediction does not depend on time, it can predict the plant response over and specified time horizon in dependency on MVs. The total numbers of 3256 samples of measured data have been applied from the experiment. For the training, $80 \%$ of the experiment was used, i.e., 2605 samples. Remaining $20 \%$ was used for the test. In this paper, the underground temperature and syngas calorific value have been modeled according to Table 1. The behavior of input observations is shown in Figure 7. A comparison of predicted and measured targets is shown in Figures 8 and 9. The test was performed on new data. The quality of prediction was evaluated based on several common statistical measures, i.e., the mean square error (MSE) (17), coefficient of correlation $\left(r_{y Y}\right)(18)$, root mean squared error (RMSE) (19), and the relative root mean squared error (RRMSE) (20):

$$
\begin{aligned}
& \text { MSE }=\frac{1}{N} \sum_{i=1}^{N}\left(Y_{i}-y_{i}\right)^{2}, \\
& r_{y Y}=\frac{\sum_{i=1}^{N}\left(Y_{i}-\bar{Y}\right)\left(y_{i}-\bar{y}\right)}{\sqrt{\sum_{i=1}^{N}\left(Y_{i}-\bar{Y}\right)^{2}} \sqrt{\sum_{i=1}^{N}\left(y_{i}-\bar{y}\right)^{2}}}, \\
& \operatorname{RMSE}=\sqrt{\frac{1}{N} \sum_{i=1}^{N}\left(Y_{i}-y_{i}\right)^{2}}, \\
& \operatorname{RRMSE}=\frac{\sqrt{\frac{1}{N} \sum_{i=1}^{N}\left(Y_{i}-y_{i}\right)^{2}}}{\frac{1}{N} \sum_{i=1}^{N} y_{i}} \times 100 .
\end{aligned}
$$

Variable $y$ represents the measured target, and $Y$ its prediction. In control, $Y$ can be the desired value. Variable $\bar{y}$ represents the average of target values $y_{i}$ and $\bar{Y}$ is the average of the predicted value $Y_{i}(i=1, \ldots, N)$. Parameter $N$ represents the number of samples in the training or testing set. 
Figure 7

Observations obtained from UCG experiment (i.e., manual control)

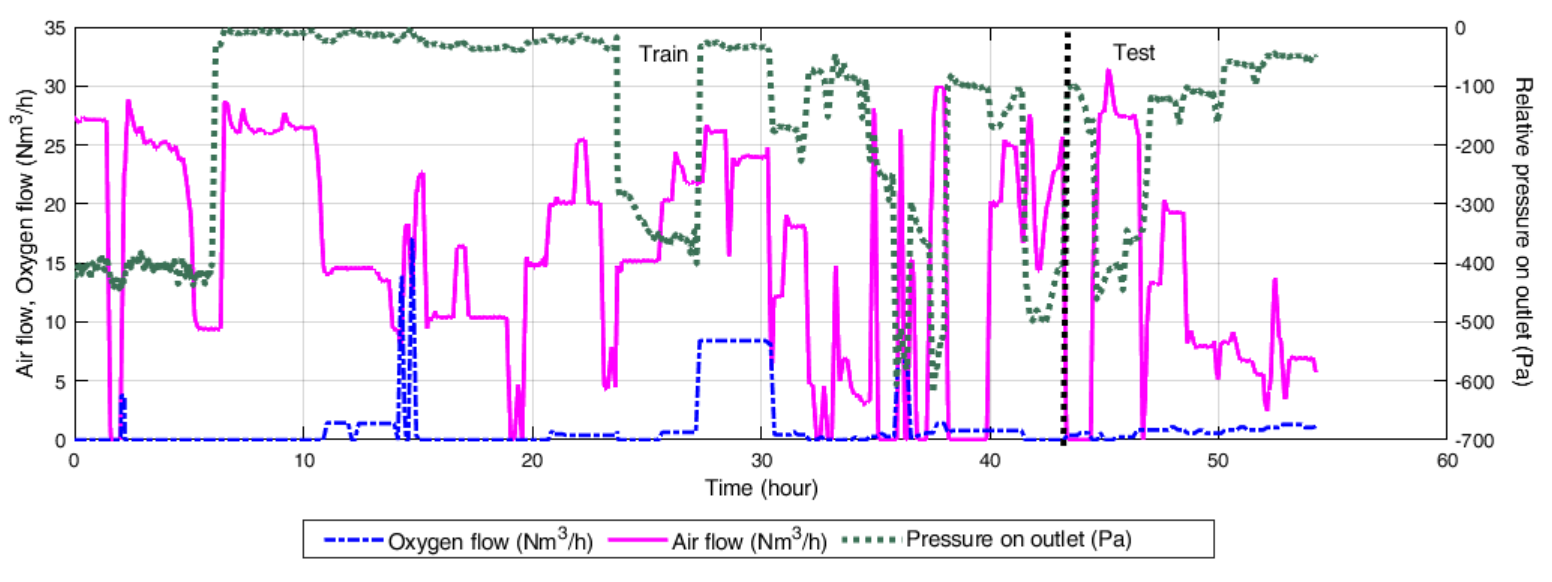

\section{Figure 8}

Measured and simulated calorific value by MARS model with three input observations

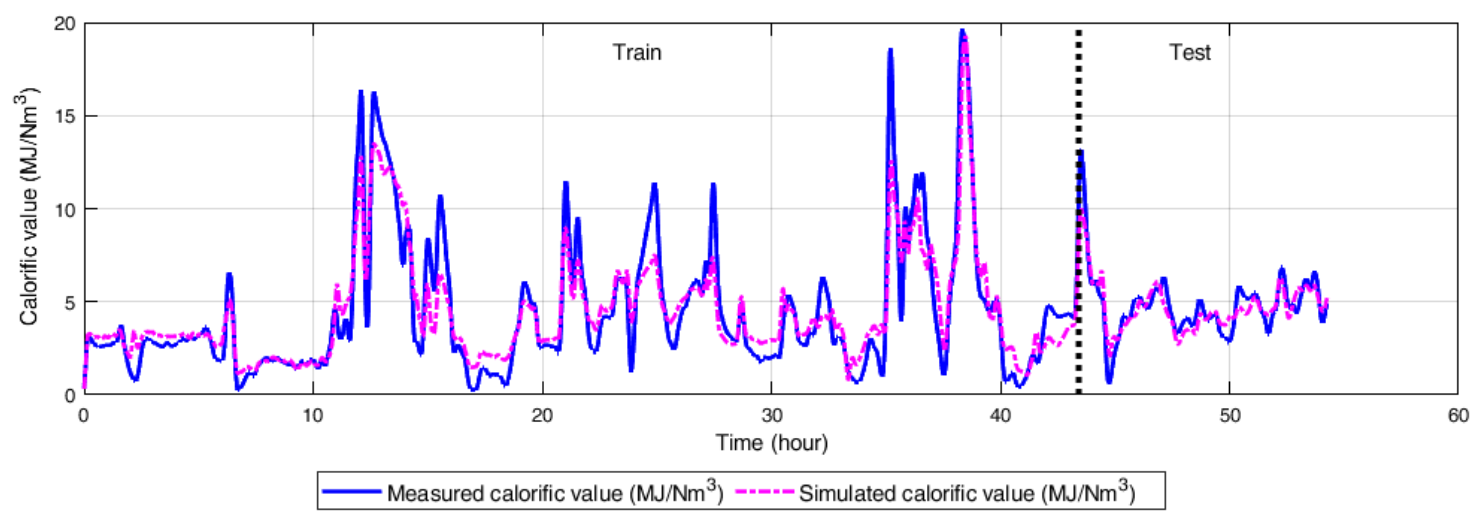

\section{Figure 9}

Measured and simulated temperature by MARS model with three input observations

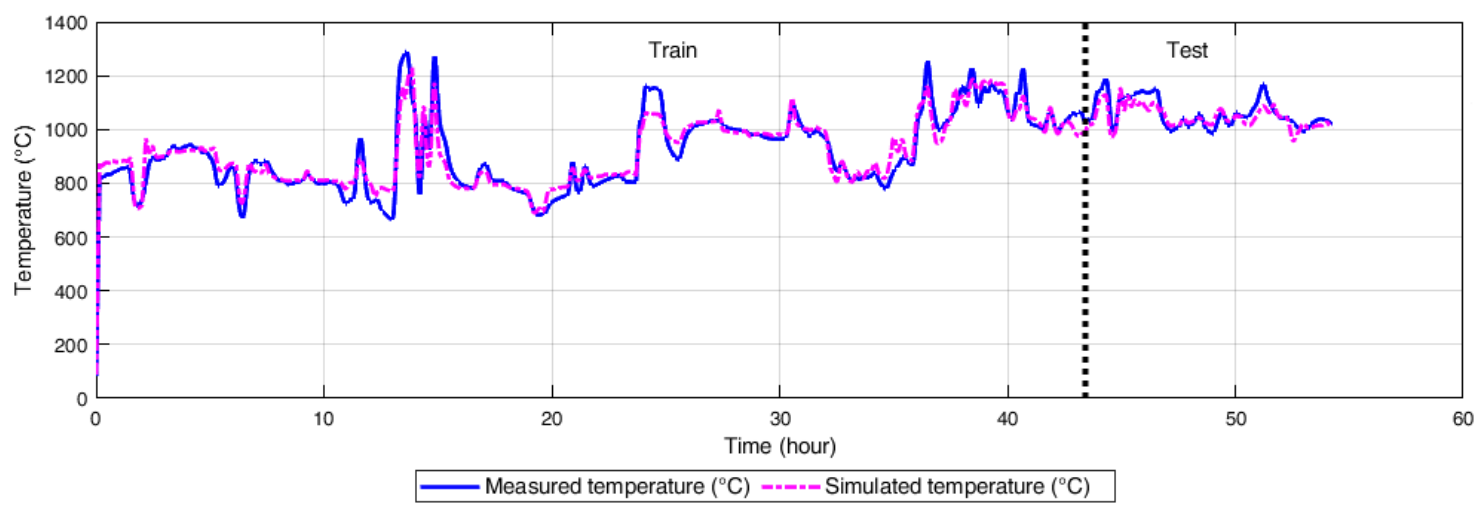


Table 2

A comparison of modeling methods based on machine learning (division on training and testing)

\begin{tabular}{|c|c|c|c|c|c|c|c|c|c|}
\hline \multirow{2}{*}{ Observations } & \multirow{2}{*}{$\begin{array}{c}\text { Target } \\
\text { variable }\end{array}$} & \multicolumn{4}{|c|}{ Training } & \multicolumn{4}{|c|}{ Test } \\
\hline & & MSE & $r_{y Y}$ & RMSE & $\begin{array}{l}\text { RRMSE } \\
(\%)\end{array}$ & MSE & $r_{y Y}$ & RMSE & $\begin{array}{l}\text { RRMSE } \\
(\%)\end{array}$ \\
\hline$u_{1}, u_{2}$ & $y_{1}$ & 7.2259 & 0.6868 & 2.6881 & 59.8328 & 11.8804 & 0.3767 & 3.4468 & 71.6223 \\
\hline$u_{1}, u_{2}, u_{3}$ & $y_{1}$ & 6.2375 & 0.7375 & 2.4975 & 55.5903 & 4.0252 & 0.5080 & 2.0063 & 41.6896 \\
\hline$u_{1}, u_{2}$ & $y_{2}$ & 9289.4151 & 0.7091 & 96.3816 & 10.5519 & 19952.8736 & 0.2149 & 141.2546 & 13.3129 \\
\hline$u_{1}, u_{2}, u_{3}$ & $y_{2}$ & 5040.1402 & 0.8545 & 70.9939 & 7.7725 & 10359.7920 & 0.2653 & 101.7831 & 9.5928 \\
\hline
\end{tabular}

These measures were evaluated in the training and testing phase (see Table 2). The results show that better prediction performance can be reached when three input observations were used. These observations will be used as manipulated variables in MPC.

\section{Table 3}

Basis function of piecewise linear type MARS model of calorific value

\begin{tabular}{l|l}
\hline Basis function & \multicolumn{1}{c}{ Equation } \\
\hline $\mathrm{BF} 1$ & $\max \left(0,1.4-u_{2}\right)$ \\
\hline $\mathrm{BF} 2$ & $\max \left(0,3.209-u_{1}\right)$ \\
\hline $\mathrm{BF} 3$ & $\max \left(0, u_{3}+48.712\right)$ \\
\hline $\mathrm{BF} 4$ & $\max \left(0, u_{1}-3.209\right) \times \max \left(0, u_{3}+296.53\right)$ \\
\hline $\mathrm{BF} 5$ & $\mathrm{BF} 3 \times \max \left(0, u_{2}-0.5\right)$ \\
\hline $\mathrm{BF} 6$ & $\mathrm{BF} 3 \times \max \left(0,0.5-u_{2}\right)$ \\
\hline $\mathrm{BF} 7$ & $\max \left(0, u_{2}-1.45\right)$ \\
\hline $\mathrm{BF} 8$ & $\max \left(0,1.45-u_{2}\right)$ \\
\hline $\mathrm{BF} 9$ & $\mathrm{BF} 2 \times \max \left(0, u_{2}-0.34\right)$ \\
\hline $\mathrm{BF} 10$ & $\mathrm{BF} 2 \times \max \left(0,0.34-u_{2}\right)$ \\
\hline $\mathrm{BF} 11$ & $\mathrm{BF} 9 \times \max \left(0, u_{3}+102.58\right)$ \\
\hline $\mathrm{BF} 12$ & $\mathrm{BF} 9 \times \max \left(0,-102.58-u_{3}\right)$ \\
\hline $\mathrm{BF} 13$ & $\mathrm{BF} 9 \times \max \left(0,-87.928-u_{3}\right)$ \\
\hline $\mathrm{BF} 14$ & $\mathrm{BF} 8 \times \max \left(0, u_{3}+249.07\right)$ \\
\hline $\mathrm{BF} 15$ & \\
\hline
\end{tabular}

The calorific value will be used as the only one output variable that will be stabilized on the desired setpoint, i.e., the MISO system is regarded. The model of temperature will provide just information about the reached temperature at given manipulated variables. Equation (21) represents the piecewise linear type of MARS model for the prediction of caloric value:

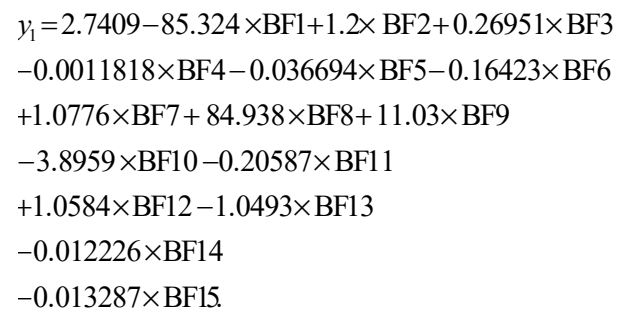

Table 3 shows the calculation of basis functions for the given model. The model (21) can be transformed into linear time-invariant (LTI) discrete state-space form by further linearization and used as an internal predictive model of MPC controller.

Similarly, Equation (22) along with Table (4) represent the MARS model for the prediction of underground temperature. The MARS models (21) and (22) will be used in an adaptive MPC scheme for the UCG imitation.

$$
\begin{aligned}
& y_{2}=835.76+12.49 \times \mathrm{BF} 1-25.957 \times \mathrm{BF} 2-2.4513 \times \mathrm{BF} 3 \\
& -16.838 \times \mathrm{BF} 4-30.646 \times \mathrm{BF} 5+232.27 \times \mathrm{BF} 6 \\
& +14.128 \times \mathrm{BF} 7+0.039049 \times \mathrm{BF} 8+0.27664 \times \mathrm{BF} 9 \\
& -209.44 \times \mathrm{BF} 10-722.08 \times \mathrm{BF} 11+0.20386 \times \mathrm{BF} 12 \\
& +18.433 \times \mathrm{BF} 13+54.165 \times \mathrm{BF} 14-0.022901 \times \mathrm{BF} 15 \\
& -3.1304 \times \mathrm{BF} 16-177.4 \times \mathrm{BF} 17 .
\end{aligned}
$$




\section{Table 4}

Basis function of piecewise linear type MARS model of temperature value

\begin{tabular}{|c|c|}
\hline Basis function & Equation \\
\hline $\mathrm{BF} 1$ & $\max \left(0, u_{2}-0.77\right)$ \\
\hline $\mathrm{BF} 2$ & $\max \left(0,0.77-u_{2}\right)$ \\
\hline BF3 & $\max \left(0, u_{3}+97.921\right)$ \\
\hline $\mathrm{BF} 4$ & $\mathrm{BF} 2 \times \max \left(0, u_{1}-15.163\right)$ \\
\hline BF5 & $\mathrm{BF} 2 \times \max \left(0,15.163-u_{1}\right)$ \\
\hline BF6 & $\max \left(0, u_{1}-27.035\right)$ \\
\hline $\mathrm{BF} 7$ & $\max \left(0,27.035-u_{1}\right)$ \\
\hline BF8 & $\mathrm{BF} 4 \times \max \left(0, u_{3}+412.11\right)$ \\
\hline BF9 & $\mathrm{BF} 4 \times \max \left(0,-412.11-u_{3}\right)$ \\
\hline BF10 & $\mathrm{BF} 6 \times \max \left(0, u_{2}-0.25\right)$ \\
\hline BF11 & $\mathrm{BF} 6 \times \max \left(0,0.25-u_{2}\right)$ \\
\hline $\mathrm{BF} 12$ & $\mathrm{BF} 7 \times \max \left(0, u_{3}+22.772\right)$ \\
\hline $\mathrm{BF} 13$ & $\max \left(0, u_{1}-14.569\right)$ \\
\hline $\mathrm{BF} 14$ & $\max \left(0,14.569-u_{1}\right) \times \max \left(0, u_{3}+16.44\right)$ \\
\hline $\mathrm{BF} 15$ & $\max \left(0,14.569-u_{1}\right) \times \max \left(0,-16.44-u_{3}\right)$ \\
\hline BF16 & $\mathrm{BF} 14 \times \max \left(0, u_{2}-1.4\right)$ \\
\hline BF17 & $\mathrm{BF} 14 \times \max \left(0,1.4-u_{2}\right)$ \\
\hline
\end{tabular}

\subsection{Adaptive MPC Results}

The UCG process is strongly nonlinear, or its properties can be changed with time. Concerning calorific value and underground temperature variations, this process can be unstable when the change occurs from one operating condition to another. The UCG is typically regarded as a MIMO system, but in our research, only MISO system was investigated.

During MPC, the future plant behavior is predicted by linear model plant output. Due to the fact that this prediction may not be accurate, the goal is to tune the MPC to be as less sensitive to predictive errors as possible (i.e., the robustness of the controller is achieved). In nonlinear systems controlled by conventional MPC, the LTI prediction accuracy can be degraded, and MPC performance becomes unacceptable. Furthermore, the MPC proposed in specific operating condition does not give sufficient control efficiency over a wide operating range. Adaptive MPC can solve this problem by updating the prediction model for changing operating conditions. The optimizer performs QP minimization (13) with a given number of iterations to optimize control moves. Figure 10 shows the basic concept of adaptive MPC proposed for UCG. In the proposal, a linearized version of the MARS model (21) at the current operating point has been used. In linearization, the gradient of the nonlinear function is taken representing all variables, and linear representation at that point is created. It enables us

Figure 10

The basic concept of adaptive MPC applied to UCG

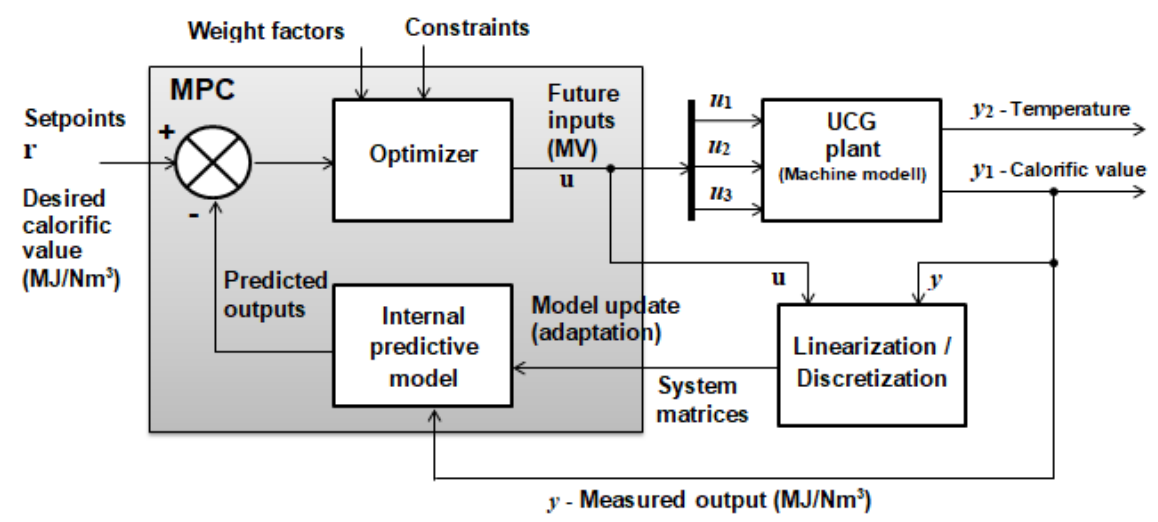


to put the model into a linear state-space form. Discrete-state-space MISO model of UCG obtained from MARS is the following:

$$
\begin{aligned}
& x(k+t)=\mathbf{A} x(k)+\mathbf{B u}(k), \\
& y(k)=\mathbf{C} x(k)+\mathbf{D u}(k), \\
& \mathbf{A}=1, \mathbf{B}=\left(\begin{array}{ll}
-0.2460 & 7.6454
\end{array}\right), \\
& \mathbf{C}=1, \mathbf{D}=\left(\begin{array}{ll}
0 & 0
\end{array}\right) .
\end{aligned}
$$

Equation (23) represents the resulting linear-time-invariant (LTI) model of calorific value that can be affected by two manipulated variables. Resulting LTI model for three manipulated variables represents the following equations:

$$
\begin{aligned}
& x(k+t)=\mathbf{A} x(k)+\mathbf{B u}(k), \\
& y(k)=\mathbf{C} x(k)+\mathbf{D u}(k), \\
& \mathbf{A}=1, \mathbf{B}=\left(\begin{array}{lll}
0.0623 & 11.97 & 0.08483
\end{array}\right), \\
& \mathbf{C}=1, \mathbf{D}=\left(\begin{array}{lll}
0 & 0 & 0
\end{array}\right) .
\end{aligned}
$$

The adaptive MPC estimates its prediction model states via linear-time-varying Kalman filter because the prediction model parameters are changing at run time. In adaptive MPC, the prediction model (i.e., the internal controller's model) is continually updated as operating condition change. A sampling time $T_{s}=1 \mathrm{~s}$ has been applied. The data from the model are continually estimated within the smallest sampling period $T_{s} / 5=0.2 \mathrm{~s}$ by recursive polynomial estimator and new ARX model (25) is calculated at the main sampling period $T_{s}$. The new ARX model is converted into statespace at the sampling period $T_{s}=1 \mathrm{~s}$. This model then updates the controller's internal prediction model. Recursive polynomial estimation of ARX-MISO model considers the following model structure:

$$
\mathbf{A}(q) y(k)=\mathbf{B}(q) \mathbf{u}\left(k-n_{k}\right)+e(k),
$$

where $y(k)$ is the output at time step $k$; $q$ is the timeshift operator, and $n_{k}$ is the input delay; $\mathbf{u}(k)$ represents the vector of MVs and $e(k)$ represents whitenoise disturbance value. For MISO systems, there are as many $\mathbf{B}(q)$ polynomials as the number of inputs.

The objective of the control is to maintain the calorific value of syngas at its reference values when UCG runs. The internal plant model is continually updated at each control interval to obtain nonlinear control of the UCG plant. Adaptive MPC continuously updates the plant model and nominal conditions at each sampling period. Nominal conditions and plant model stay fixed over the prediction horizon. The online estimation is used in the closed-loop. Conventional MPC regards nominal operation point at which the nonlinear plant model is linearized. In adaptive MPC, as time evolves, this nominal point should be updated to be consonant with the updated plant model.

The UCG plant model can be written in term of deviation from the nominal conditions:

$$
\begin{aligned}
& \mathbf{x}(k+1)=\overline{\mathbf{x}}+\mathbf{A}(\mathbf{x}(k)-\overline{\mathbf{x}})+\mathbf{B}\left(\mathbf{u}_{\mathbf{t}}(k)-\overline{\mathbf{u}}_{\mathbf{t}}\right)+\Delta \overline{\mathbf{x}}, \\
& \mathbf{y}(k)=\overline{\mathbf{y}}+\mathbf{C}(\mathbf{x}(k)-\overline{\mathbf{x}})+\mathbf{D}\left(\mathbf{u}_{\mathbf{t}}(k)-\overline{\mathbf{u}}_{\mathbf{t}}\right) .
\end{aligned}
$$

In Equation (26), the general MIMO system structure with parameter matrices $\mathbf{A}, \mathbf{B}, \mathbf{C}$, and $\mathbf{D}$ has been considered. These matrices are continually updated. Parameter $\mathbf{u}_{\mathrm{t}}$ represents the vector of combined plant input variables, comprising the measured and unmeasured disturbance. Variables $\overline{\mathbf{x}}, \Delta \overline{\mathbf{x}}, \overline{\mathrm{u}}_{\mathrm{t}}$ and $\bar{y}$ represent $n_{x}$ nominal states, $n_{x}$ nominal state increments, $n_{u t}$ nominal MVs and $n_{y}$ nominal MOs which are updated.

In the simulation with MPC, no disturbance was used, only the noise was added to the manipulated variables and output variables to simulate real conditions. In the result, it can be seen how the setpoint is tracked with the measured plant output. The prediction horizon was set up to $10 \mathrm{~s}$, control horizon to $2 \mathrm{~s}$. The piecewise linear type of MARS model has been linearized to obtain discrete-state-space matrices for internal prediction model of MPC. The linearized plant model is joined with the MPC controller. The nominal values are also set up in the MPC controller. An initial plant model initializes online polynomial estimation. In this case, the Kalman filter is used for recursive online estimation of the autoregressive model (ARX). The resulting discrete-time ARX model is converted into a discrete state-space form with the same order and sample time as the original plant model has.

Simulations were performed in Matlab-Simulink environment with the support of Model Predictive Control Toolbox [41]. In Figure 11, the simulation model to simulate adaptive MPC with three manipulation variables is proposed. A simulation model with one manipulation variable was also proposed to compare MPC with PI controller. For the UCG plant imitation, 


\section{Figure 11}

Matlab-Simulink block scheme for adaptive MPC simulation (modified after [6])

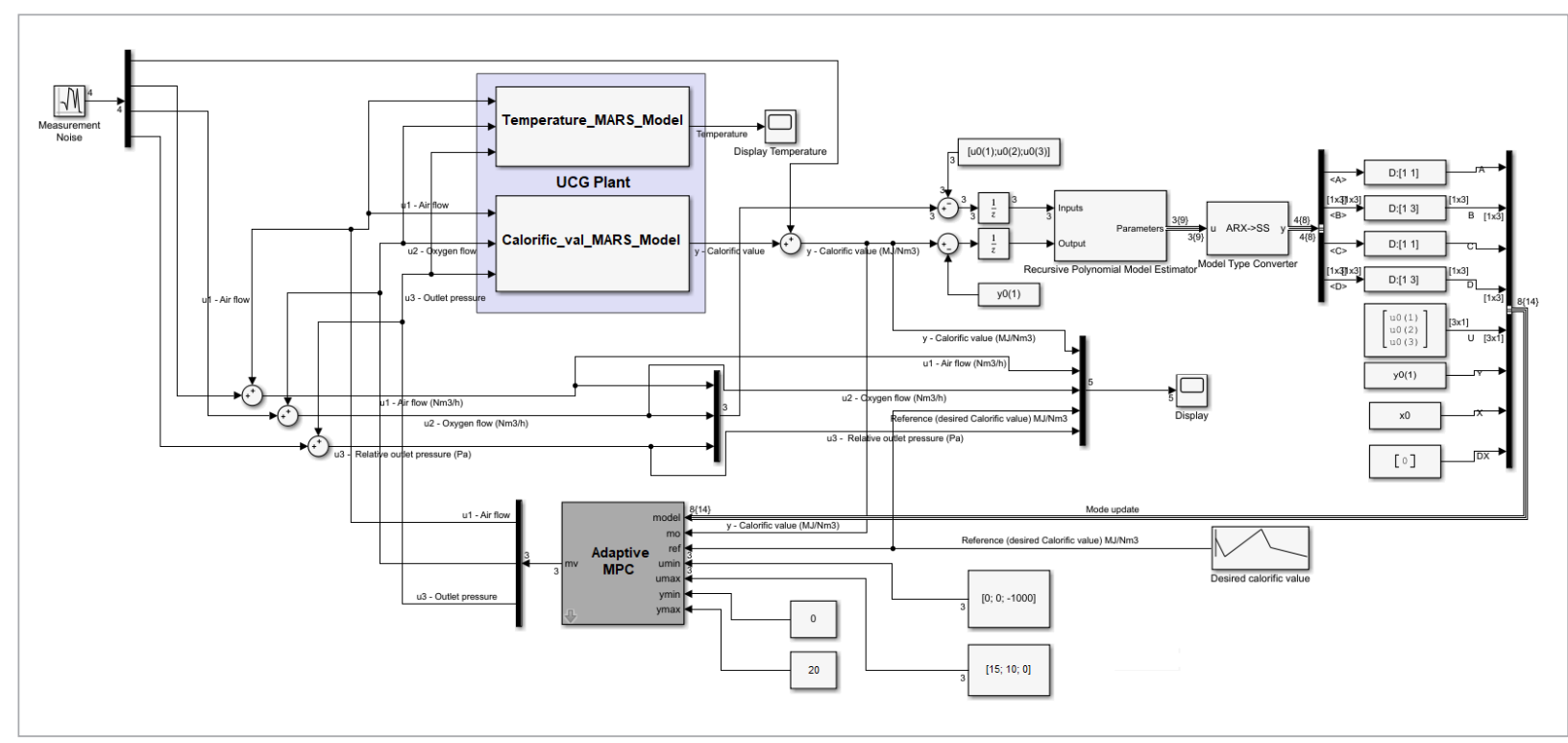

Level 2 Matlab S-Functions were used in the control scheme. These S-Functions implement MARS models. The discrete state space-model derived from ARX model is decomposed into relevant real-valued matrices, i.e., $\mathbf{A}$ (square state matrix with a dimension of $n_{x} \times n_{x}$ ), B (input-to-state matrix with a dimension of $n_{x} \times n_{u}$ ), $\mathbf{C}$ (state to output matrix with a dimension of $n_{y} \times n_{u}$ ), $\mathbf{D}$ (feedthrough matrix with as many rows as outputs, and as many columns as inputs), U, Y, X, and DX to update original internal prediction model of the MPC controller, where $n_{x}$ represents the number of plant model states; $n_{u}$ represents the number of plant MVs, and $n_{y}$ represents the number of plant MOs. When the ARX state-space model is used in the conversion, only A and B matrices are updated (e.g., for comparison the ARMAX model has matrices A, B, and C). Vector $\mathbf{X}$ of the length $n_{x}$ replaces nominal states in the internal prediction model of the MPC controller. Vector $\mathbf{Y}$ of length $n_{y}$ replaces nominal outputs in the internal prediction model of the MPC block. Vector $\mathrm{U}$ of length $n_{w}$, replaces nominal inputs in the internal prediction model of the MPC block. Vector DX of length $n_{x}$, replaces nominal $\Delta \mathbf{x}$ in the internal prediction model of MPC block. For discrete-time models, $\mathbf{D} \mathbf{X}=\mathbf{x}(k+1)-\mathbf{x}(\mathrm{k})=f(\mathbf{X}, \mathbf{U})-\mathbf{X}$, i.e., $\mathbf{D} \mathbf{X}=f(\mathbf{X}, \mathbf{U})$. Vectors $\mathbf{X}$, $\mathbf{Y}$, and $\mathbf{U}$ represent plant state, output, and input at the operating point.
In the first simulation, the comparison of stabilization of calorific value to various setpoints by the classical discrete PI controller and MPC was performed (see Figure 12). The discrete PI controller is well applicable in slow and delayed processes. This type of controller has already been applied in UCG to stabilize injected airflow, underground temperature, a ratio of $\mathrm{CO} /\left(\mathrm{CO}+\mathrm{CO}_{2}\right)$ and oxygen concentration in syngas [24]. Since it would be complicated to create a decomposed PI control for all MVs, only one MV was considered in simulation, i.e., $u$ - air flow. To compare the performance of MPC with the PI controller, the MPC controller also worked only with one manipulating variable. The discrete PI controller that was applied has the following form:

$$
\begin{aligned}
& \Delta u(k)=u(k)-u(k-1) \\
& =K_{P}\left[e(k)+\left(\frac{T_{s}}{T_{I}}-1\right) e(k-1)\right]=K_{P} e(k)-K_{P}\left(1-\frac{T_{s}}{T_{I}}\right) e(k-1) \\
& =q_{0} e(k)+q_{1} e(k-1)=0.038429 e(k)-0.031467 e(k-1),
\end{aligned}
$$

where $\Delta u(k)$ is an increase of the manipulating variable $u$ (i.e., injected air flow), $k$ is an index of the control period, $u(k)$ is the value of manipulating variable 
Figure 12

A comparison of calorific value stabilization when stabilizing by MPC and PI controller

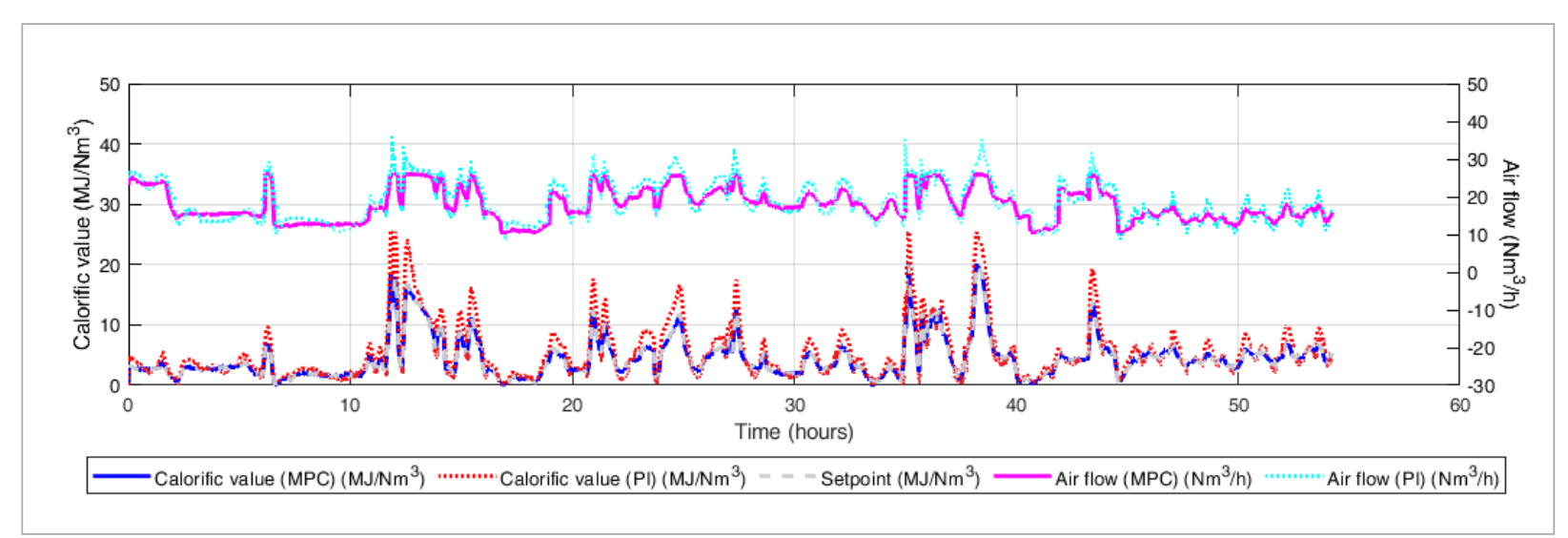

in step $k, e(k)$ is the control error $(e(k)=w(k)-y(k))$, $w(k)$ is the setpoint in step $k$ (i.e., desired calorific value), $q_{0}, q_{1}$ are defined parameters of the resultant discrete controller by the following substitution: $q_{0}=K_{P}$, $q_{1}=-K_{P}\left(1-T_{s} / T_{I}\right), T_{s}$ is the sampling period. $K_{P}$ is the proportional gain and $T_{I}$ is integral time (i.e., constant of integration).

The discrete PI controller was designed by the Modified Ziegler-Nichol method, which is based on the ARX model $[10,24]$. The simulation of the calorific value stabilization through the PI and MPC controller was performed for the entire UCG experiment. Discrete PI controller continually calculated increments $\Delta u$ of one gasification agent (i.e., air) to stabilize the syngas calorific value, i.e., variable $y$. The experiment used to simulate MPC lasted $195300 \mathrm{~s}$, which is over 54 hours. The MARS model that was applied was trained in an experiment using only air as a gasification agent.

The desired calorific value was continually set according to the UCG manual control. For the performance evaluation, the MSE (17) was used. As expected, stabilization of calorific value by MPC has reached a lower MSE (i.e., MSE=0.0552) than in PI control (i.e., MSE $=5.6025)$. The upper limit for the manipulation variable in the case of MPC was set to $35 \mathrm{Nm}^{3} / \mathrm{h}$. This setting was based on the assumption that more air (i.e., atmospheric oxygen) is needed to support gasification chemical reactions since technical oxygen was not added. In stabilizing the syngas calorific value by the discrete PI controller, a higher overshooting and a longer control time can also be observed. It can be stated that the MPC was faster than the discrete PI controller. Next, the simulation with three MVs was performed.

In the simulation with three manipulation variables, constraints in MPC were set up as follows:

$y_{1, \text { min }}=0 \mathrm{MJ} / \mathrm{Nm}^{3}, y_{1, \text { max }}=20 \mathrm{MJ} / \mathrm{Nm}^{3}$,

$u_{1, \text { min }}=0 \mathrm{Nm}^{3} / \mathrm{h}, u_{1, \text { max }}=15 \mathrm{Nm}^{3} / \mathrm{h}$,

$u_{2, \min }=0 \mathrm{Nm}^{3} / \mathrm{h}, u_{2, \max }=10 \mathrm{Nm}^{3} / \mathrm{h}$,

$u_{3, \text { min }}=-1000 \mathrm{~Pa}, u_{3, \max }=0 \mathrm{~Pa}$.

The desired calorific value for MPC was the same as the measured calorific value from the manual control of the UCG. Simulation results show that the MPC gives less air and more oxygen (i.e., as optimized manipulation variables) during the experimental UCG (see Figures 13 and 14) in comparison with manual control (see Figures 7 and 8). More air injected into the reactor can cause heat loss because cold air cools the coal that is gasified. Atmospheric air itself contains less oxygen and more nitrogen, and it needs to be supplied more oxygen to support combustion reactions with carbon. The pure technical oxygen that was blown as an auxiliary oxidant has a more significant influence on exothermic reactions (i.e., chemical reactions that releases energy through light or heat). Usually, only industrial oxygen and water vapor are used in real industrial UCG. In our case, the steam was not used because of a suitable water vapor generator was no available. In addition, the temperature of the overheated vapor at a higher pressure (i.e., up to 1 bar) would have to be at least $120^{\circ} \mathrm{C}$. MPC has also reduced outlet pressure, 
Figure 13

The behavior of the measured calorific value and its reference values during simulated MPC of UCG

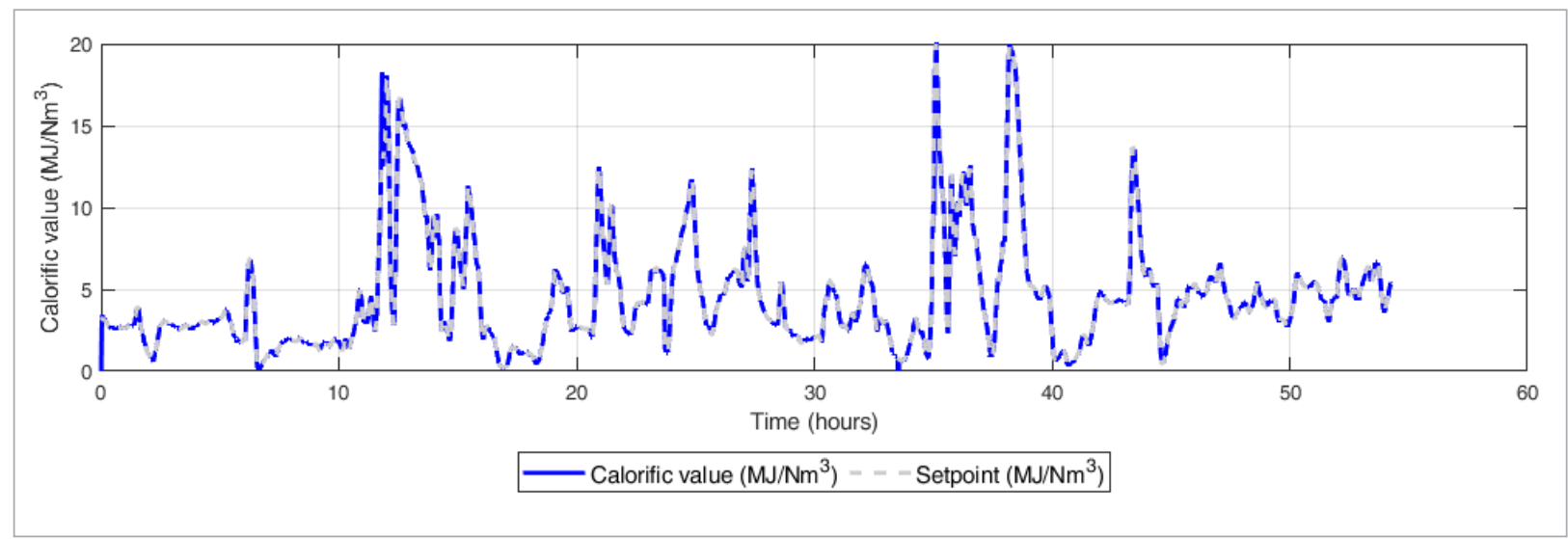

Figure 14

The behavior of the optimized manipulation variables during simulated MPC of UCG

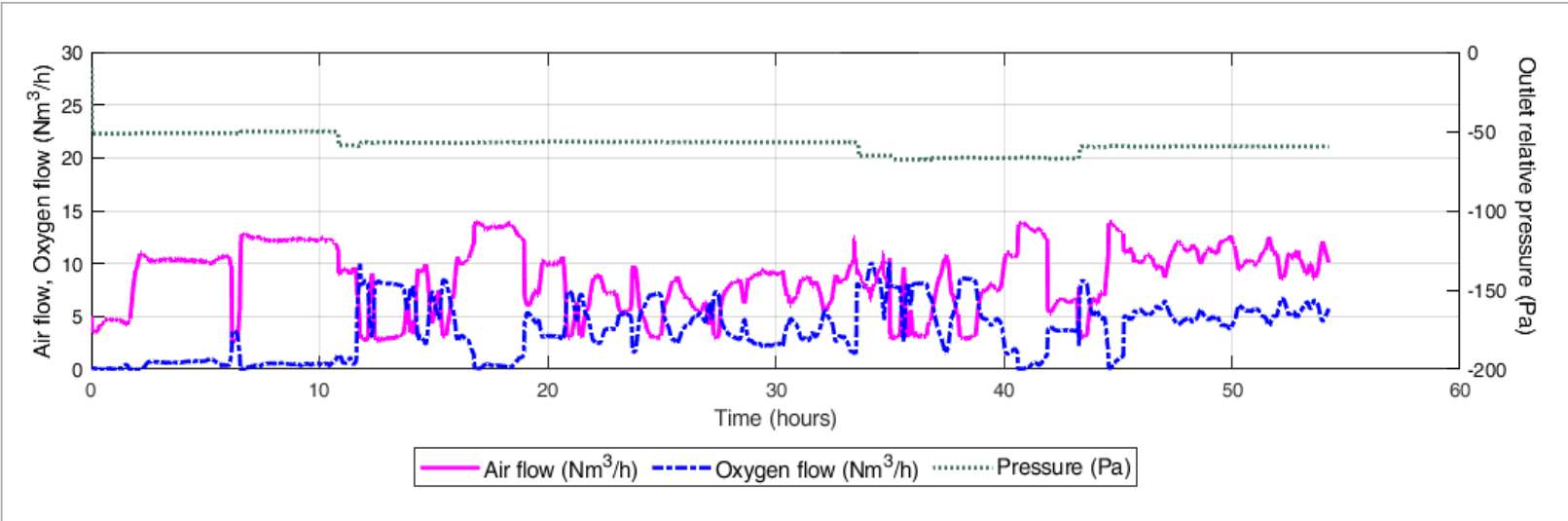

Figure 15

Modeled temperature during simulated MPC of UCG

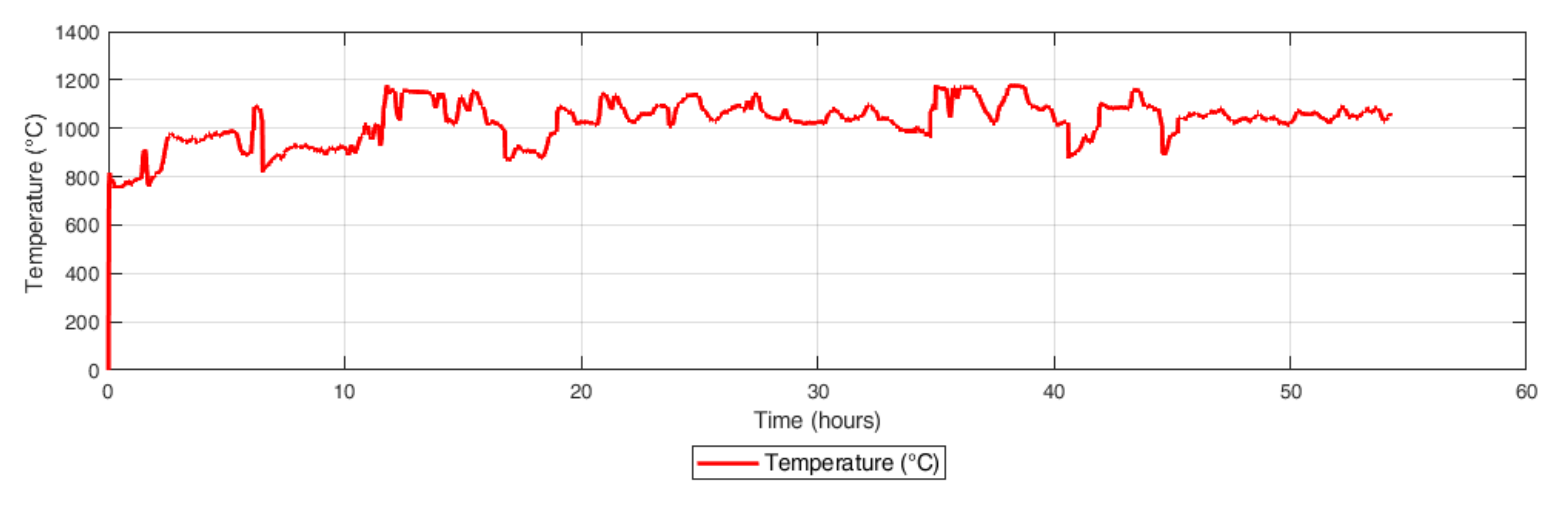


which means that less electric power would be needed for exhausting. More suction at the outlet causes the other air to be drawn into the gasification reactor through various gaps and cracks in the overburden rocks. However, it should be noted, that there exist UCG technologies where gasification is controlled only by a regulated underpressure using an exhaust fan without direct injection of the inlet oxidant. The air enters into the underground reactor by suction through the air well, and through the various joints and cracks in the overburden. The advantage of this technology is that there are no gas leaks from underground to surface.

In MPC, the calorific value was well stabilized to its setpoints without significant overshoots. It can be seen that there was also a low variability in some manipulated variables (i.e., airflow and outlet pressure). However, this was achieved by defining constraints on manipulation variables. With less variance in manipulating variables, there is also less mechanical stress on the actuator. By this way, the underground temperature can be longer maintained over $1000^{\circ} \mathrm{C}$ (see Figure 15), allowing a more calorific syngas to be produced. It should be noted that higher temperatures above $1000^{\circ} \mathrm{C}$ and higher syngas calorific values are typically achieved with oxygen UCG technologies. On the other hand, more oxygen injected into the underground reactor can be more expensive. In manual control, the temperature was approximately $1000^{\circ} \mathrm{C}$, and for a short time, it reached approximately $1300^{\circ} \mathrm{C}$ (see Figure 9).

Since thermocouples cannot be used in real UCG (i.e., thermocouple holes could cause pressure losses), the estimation of the temperature from the advanced regression model has been proposed. In this paper, the MARS model was used because of its more straightforward implementation for MPC simulation. It was found that MARS model well models non-linearities in the UCG data. In another work (e.g., [25]), a machine model based on the Support Vector Regression (SVR) was successfully applied by the author of this paper. The underground temperature can be estimated based on control variables or by inverse way from the measured syngas composition.

\section{Conclusion}

In this paper, an adaptive MPC applied to UCG was investigated. The UCG has the potential to be used on unmined or deepest coal seams, as an alternative for traditional coal mining with less environmental impacts. The advanced control of this process is very complicated as the underground gasifier crosses several stages, where operating conditions are changing. The UCG experiment was performed to acquire data for modeling and control simulation. Two MARS models for UCG plant imitation (i.e., a model for syngas calorific value and underground temperature model) were designed in the paper. The linearized discrete state-space version of the calorific value model was used as an internal prediction model of the MPC. The MPC with constraints showed performance improvement when compared with manual control in terms of manipulation variables optimization. This approach to UCG control method can completely replace the manual control as it is based on a predictive model that is continuously adapted to changing operating conditions. The simulations results have shown that MPC can continually optimize the manipulation variables to maintain the desired calorific value. Some optimized manipulated variables have lower variability in comparison with manual control. In addition, optimized manipulation variables can maintain a temperature over $1000^{\circ} \mathrm{C}$ for a long time that is essential for the producing calorific elements of the syngas (i.e., CO). Moreover, MPC has reduced the underpressure at the outlet, so the intake of free air into the gasification reactor can be eliminated. It has also been presented that MPC achieves better performance than the traditional discrete PI controller. At the same time, MPC can eliminate control error faster without significant overshoots. However, it must be noted that the system decomposition for the PI control by several manipulations variables was not performed in this work, and only the airflow was used to stabilize the calorific value. The results show the potential of the proposed control method. The results can serve as a basis for the application of MPC for the advanced control of in-situ UCG. Further research will focus on implementing MPC on automation hardware and software (i.e., PLC + SCADA/ $\mathrm{HMI}$ ) and verifying the control system online during experimental gasification.

\section{Acknowledgment}

This work was supported by the Slovak Grant Agency for Science under grant VEGA 1/0273/17, and by the Slovak Research and Development Agency under the contract No. APVV-14-0892 and contract No. APVV-18-0526. 


\section{References}

1. Abdel-Hadi, E. A. A., Hsu, T. R. Computer Modeling of Fixed Bed Underground Coal Gasification Using the Permeation Method. Journal of Energy Resources Technology, 1987, 109, 11-20. https://doi. org/10.1115/1.3231316

2. Albertos, P., Ortega, R. On Generalized Predictive Control: Two Alternative Formulations. Automatica, 1989, 25(5), 753-755. https://doi.org/10.1016/00051098(89)90031-9

3. Al Seyab, R. K., Cao, Y. Nonlinear Model Predictive Control for the ALSTOM Gasifier. Journal of Process Control, 2006, 16(8), 795-808. https://doi.org/10.1016/j. jprocont.2006.03.003

4. Batenburg, D. Heat and Mass Transfer During Underground Coal Gasification. PhD Thesis, Dietz Laboratory, Delft University of Technology, 1992.

5. Beizen, E. N. J. Modeling Undeground Coal Gasification. PhD Thesis, Delft University of Technology, the Netherlands, 1996.

6. Bemporad, A., Ricker, N. L., Morari, A. Model Predictive Control ToolboxTM, User's Guide. MathWorks Inc., 2018.https://www.mathworks.com/help/pdf_doc/ $\mathrm{mpc} / \mathrm{mpc} \_$ug.pdf

7. Bequette, B. W., Mahapatra, P. Model Predictive Control of Integrated Gasification Combined Cycle Power Plants. Report, Office of Scientific and Technical Information (OSTI), 2010. https://doi.org/10.2172/1026486

8. Bliek, A., Lont, J. C., Swaaij, W. P. M. V. Gasification of Coal-Derived Chars in Synthesis Gas Mixtures Under Intraparticle Mass-Transfer-Controlled Conditions. Chemical Engineering Science, 1986, 41(7), 1895-1909. https://doi.org/10.1016/0009-2509(86)87069-5

9. Blinderman, M. S., Klimeko, A. Y. Introduction to Underground Coal Gasification and Combustion, Underground Coal Gasification and Combustion. Woodhead Publishing Series in Energy, 2018, 1-8. https://doi. org/10.1016/B978-0-08-100313-8.00001-3

10. Bobál, V., Böhm, J., Fessl, J., Machácek, J. Digital Self-Tuning Controllers: Algorithms, Implementation and Applications (Advanced Textbooks in Control and Signal Processing). Springer-Verlag, 2005.

11. Brasseur, A., Antenucci, D., Bouquegneau, J.-M., Coëme, A., Dauby, P., Létolle, R., Mostade, M., Pirlot, P., Pirard, J.-P. Carbon Stable Isotope Analysis as a Tool for Tracing Temperature During the El Tremedal Underground Coal Gasification at Great Depth. Fuel, 2002, 81(1), 109117. https://doi.org/10.1016/S0016-2361(01)00114-4
12. Burton, E., Friedmann, J., R. Upadhye, R. Best Practices in Underground Coal Gasification. Report No. W-7405Eng-48. Technical Report, Lawrence Livermore National Laboratory, 2013.

13. Camacho, E. F., Bordons, C. Model Predictive Control, Second Edition. Springer, 200\%. https://doi. org/10.1007/978-0-85729-398-5

14. Chugh, M., Thumsi, S. S., Keshri, V. A. Comparative Study Between Least Square Support Vector Machine (LSSVM) and Multivariate Adaptive Regression Spline (MARS) Methods for the Measurement of Load Storing Capacity of Driven Piles in Cohesionless Soil. International Journal of Structural and Civil Engineering Research, EJournal Publishing, 2015, 189-194. https://doi. org/10.18178/ijscer.4.2.189-194

15. De Vries, R. A. J., Verbruggen, H. B. Advanced in Model-Based Predictive Control, Chapter Multivariable Unified Predictive Control. Oxford University Press, 1994.

16. Dobbs, R. L., Krantz, W. B. Combustion Front Propagation in Underground Coal Gasification. Final Report, Work Performed Under Grant No. DE-FG2286PC90512. Technical Report, University of Colorado, Boulder Department of Chemical Engineering, 1990. https://doi.org/10.2172/6035494

17. Durdán, M., Kostúr, K. Modeling of Temperatures by Using the Algorithm of Queue Burning Movement in the UCG Process. Acta Montanistica Slovaca, 2015 20(3), 181-191.

18. Friedman, J. H. Multivariate Adaptive Regression Splines. The Annals of Statistics, 1991, 19(1), 1-67. https://doi.org/10.1214/aos/1176347973

19. Hastie T., Tibshirani R., Friedman J. H. The Elements of Statistical Learning, 2nd Edition. Springer, 2009. https://doi.org/10.1007/b94608

20. Hou, Z., Liu, S., Yin, Ch. Local Learning-based Model-Free Adaptive Predictive Control for Adjustment of Oxygen Concentration in Syngas Manufacturing Industry. IET Control Theory \& Applications, 2015. https:// doi.org/10.1049/iet-cta.2015.0835

21. Jekabsons, G. ARESLab: Adaptive Regression Splines toolbox for Matlab/Octave. 2017. http://www.cs.rtu.lv/ jekabsons

22. Ji, P., Gao, X., Huang, D., Yang, Y. Prediction of Syngas Compositions in Shell Coal Gasification Process via Dynamic Soft-Sensing Method. Proceeding of 10th IEEE International Conference on Control and Automation 
(ICCA), IEEE, 2013, 244-249. https://doi.org/10.1109/ ICCA.2013.6565140

23. Ji, T., Shi, H. Soft Sensor Modeling for Temperature Measurement of Texaco Gasifier Based on an Improved RBF Neural Network. 2006 IEEE International Conference on Information Acquisition, IEEE, 2006, 11471151. https://doi.org/10.1109/ICIA.2006.305907

24. Kačur, J., Kostúr, K. Approaches to the Gas Control in UCG. Acta Polytechnica, Czech Technical University in Prague - Central Library, 2017, 57(3), 182-200. https:// doi.org/10.14311/AP.2017.57.0182

25. Kačur, J., Laciak, M., Durdán M., Flegner, P. Utilization of Machine Learning Method in Prediction of UCG Data. ICCC 201\%: 18th International Carpathian Control Conference, IEEE, 2017, 1-6. https://doi. org/10.1109/CarpathianCC.2017.79770411

26. Ke, W., Huang, D., Yang, F., Jiang, Y. Soft Sensor Development and Applications Based on LSTM in Deep Neural Networks. 2017 IEEE Symposium Series on Computational Intelligence (SSCI), IEEE, 2017, 1-6. https:// doi.org/10.1109/SSCI.2017.8280954

27. Khan, M. M., Mmbaga, J. P., Shirazi, A. S., Liu, Q., Gupta, R. Modelling Underground Coal Gasification: a Review. Energies, 2015, 8(11), 12603-12668. https://doi. org/10.1016/B978-0-08-100313-8.00011-6

28. Koenen, M., Bergen, F., David, P. Isotope Measurements as a Proxy for Optimising Future Hydrogen Production in Underground Coal Gasification. News in Depth. 2015. https: //www.tno.nl/media/2624/information20nid1.pdf

29. Kostúr, K. Mathematical Modeling Temperature's Fields in Overburden During Underground Coal Gasification. ICCC 2014: 15th International Carpathian Control Conference, IEEE, 2014, 248-253. https://doi. org/10.1109/CarpathianCC.2014.6843606

30. Kostúr, K., Kačur, J. Developing of Optimal Control System for UCG. Proceedings of the 13th International Carpathian Control Conference (ICCC 2012), High Tatras, Slovakia, IEEE, 2012, 28-31. https://doi.org/10.1109/ CarpathianCC.2012.6228666

31. Kostúr, K., Kačur, J. Extremum Seeking Control of Carbon Monoxide Concentration in Underground Coal Gasification. IFAC-PapersOnLine, 2017, 50(1), 1377213777. https://doi.org/10.1016/j.ifacol.2017.08.2571

32. Kostúr, K., Laciak, M. Durdán, M., Kačur, J., Flegner, P. Low-Calorific Gasification of Underground Coal with a Higher Humidity. Measurement, 2015, 63, 69-80. https://doi.org/10.1016/j.measurement.2014.12.016
33. Laciak, M., Durdán, M., Kačur, J. Proposal of the UCG Control Based on Continuous Identification. ICCC'2009. Krakow: AGH - University of Science and Technology, 2009, 395-398.

34. Lee, J. H., Morari, M., García, C. E. State-Space Interpretation of Model Predictive Control. Automatica, 1994, 30(4), 707-717. https://doi.org/10.1007/978-94011-0135-6_12

35. Li, J., Zhong, W., Cheng, H., Kong, X., Qian, F. A Data-Driven Soft Sensor Modeling for Furnace Temperature of Opposed Multi-Burner Gasifier. 2011 7th International Conference on Natural Computation, IEEE, 2011, 705-710. https://doi.org/10.1109/ ICNC.2011.6022141

36. Liu, S., Hou, Z., Yin, C. Data Driven Modeling for UGI Gasification Process via a Variable Structure Genetic BP Neural Network. 2014 International Joint Conference on Neural Networks (IJCNN), IEEE, 2014, 10711078. https://doi.org/10.1109/IJCNN.2014.6889652

37. Liu, S., Hou, Z., Yin, C. Data-Driven Modeling for FixedBed Intermittent Gasification Processes by Enhanced Lazy Learning Incorporated with Relevance Vector Machine. 11th IEEE International Conference on Control \& Automation (ICCA), IEEE, 2014, 1019-1024. https://doi.org/10.1109/ICCA.2014.6871060

38. Maciejowski, J. M., Goulart, P. J., Kerrigan, E. C. Constrained Control Using Model Predictive Control. Springer Berlin Heidelberg, 2007, 273-291. https://doi. org/10.1007/978-3-540-37010-9_9

39. Mao, F. Underground Coal Gasification (UCG): A New Trend of Supply-Side Economics of Fossil Fuels. Natural Gas Industry B, 2016, 3(4), 312-322. https://doi. org/10.1016/j.ngib.2016.12.007

40. Massaquoi, J. G. M., Riggs, J. B. Mathematical Modeling of Combustion and Gasification of a Wet Coal Slab - II Mode of Combustion, Steady State Multiplicities and Extinction. Chemical Engineering Science, 1983, 38(10), 1757-1766. https://doi.org/10.1016/00092509(83)85032-5

41. MathWorks Inc., Model Predictive Control ToolboxTM Release 2018b. The MathWorks, Inc., Natick, Massachusetts, United States, 2018.

42. Milosz, M., Murzabekov, Z., Tussupova, K., Usubaliev, S. Optimisation of Discrete Processes with Bounded Control. Information Technology and Control, Publishing House Technologija, 2018, 47(4), 684-690. https://doi. org/10.5755/j01.itc.47.4.19933

43. Morari, M. Advances in Model-Based Predictive Control, Chapter Model Predictive Control: Multivariable 
Control Technique of Choice in the 1990s? Oxford University Press, 1994.

44. Ozbek, N. S., Eker, İ. A Novel Modified Delay-Based Control Algorithm with an Experimental Application. Information Technology and Control, 2019, 48(1), 90103. https://doi.org/10.5755/j01.itc.48.1.19066

45. Perkins, G. M. P. Mathematical Modelling of Underground Coal Gasifcation. PhD Thesis, School of Materials Science and Engineering, the University of New South Wales, Australia, 2005.

46. Shah, S. L., Mohtadi, C., Clarke, D. W. Multivariable Adaptive Control without a Prior Knowledge of the Delay Matrix. Systems and Control Letters, 1987, 9, 295306. https://doi.org/10.1016/0167-6911(87)90054-5

47. Srinivas, B., Amundson, N. R. A Single-Particle Char Gasification Model. AIChE Journal, 1980, 26(3), 487496, 1980. https://doi.org/10.1002/aic.690260321

48. Thorsness, C. B., Britten, J. A. Cavsim User Manual. Technical Report UCID-21667, Lawrence Livermore National Laboratory, University of California, Berkeley, CA, 1989.

49. Thorsness, C. B., Grens, E. A., Sherwood A. A One-Dimensional Model for In-Situ Coal Combustion. Technical Report UCRL-52523, Lawrence Livermore National Laboratory, University of California, Berkeley, CA, 1978.

50. Thorsness, C. B., Kang, S.-W. A General-Purpose, Packed-Bed Model for Analysis of Underground Coal Gasification Processes. Technical Report UCID-20731, Lawrence Livermore National Laboratory, University of California, Berkeley, CA, 1986.

51. Tselykh, V. R. Multivariate Adaptive Regression Splines. Machine Learning and Data Analysis, Computing Centre of the Russian Academy of Sciences, 2012, 1(3), 272-278.

52. Uppal, A. A., Bhatti, A. I., Aamir, E., Samar, R., Khan, S. Control Oriented Modeling and Optimization of One Dimensional Packed Bed Model of Underground Coal Gasification. Journal of Process Control, 2014, 24, 269277. https://doi.org/10.1016/j.jprocont.2013.12.001

53. Uppal, A. A., Butt, S. S., Khan, Q., Aschemann, H. Robust Tracking of the Heating Value in an Underground Coal Gasification Process Using Dynamic Integral Sliding Mode Control and a Gain-Scheduled Modified Utkin Observer, Journal of Process Control, 2019, 73, 113-122. https://doi.org/10.1016/j.jprocont.2018.11.005
54. Wang, G. Application of Radon Measurement and Infrared Thermal Imaging Method in the Detecting Fire Area of Small Coal Kiln. Journal of Xi'an University of Science and Technology, 2015, 35(5), 573-578.

55. Wei, Q., Liu, D. Adaptive Dynamic Programming for Optimal Tracking Control of Unknown Nonlinear Systems with Application to Coal Gasification. Transactions on Automation Science and Engineering, IEEE, 2014, 11(4), 1020-1036. https://doi.org/10.1109/ TASE.2013.2284545

56. Wiatowski, M., Kapusta, K., Stańczyk, K. Efficiency Assessment of Underground Gasification of Orthoand Meta-Lignite: High-Pressure Ex-Situ Experimental Simulations. Fuel, 2015, 236, 221-227. https://doi. org/10.1016/j.fuel.2018.08.143

57. Winslow, A. M. Numerical Model of Coal Gasification in a Packed Bed. Technical Report UCRL-77627, Lawrence Livermore National Laboratory, University of California, Berkeley, CA, 1976.

58. Wu, J. M. Radon Distribution Under the Mine and the Application of Radon Measuring in the Monitoring of the Natural Fire Zone. Thesis, Taiyuan: Shanxi Institute of Mining and Technology, 1994.

59. Xu, Q., Li, D., Tan, W. Model Predictive Control for an IGCC Gasifier. Proceedings of the 33rd Chinese Control Conference, 2014, 77442-7746. https://doi.org/10.1109/ ChiCC.2014.6896291

60. Yan, W., Shao H., Wang, X. Soft Sensing Modeling Based on Support Vector Machine and Bayesian Model Selection. Computers \& Chemical Engineering, 2004, 28(8), 1489-1498. https://doi.org/10.1016/j.compchemeng.2003.11.004

61. Zanoli, S. M., Astolfi, G., Barboni, L. Application of a New Dataset Selection Procedure for the Prediction of the Syngas Composition of a Gasification Plant. IFAC Proceedings Volumes (8th IFAC Symposium on Advanced Control of Chemical Processes Furama Riverfront, Singapore), 2012, 45(15), 868-873. https://doi. org/10.3182/20120710-4-SG-2026.00065

62. Zhang, S., Bentsman, J., Lou, X., Neuschaefer, C. Wavelet Multiresolution Model Based Generalized Predictive Control for Hybrid Combustion-Gasification Chemical Looping process, Proceedings of 51st IEEE Conference on Decision and Control (CDC), IEEE, 2012, 24092414. https://doi.org/10.1109/CDC.2012.6425869 